\title{
Changes in the list of Finnish insects during 2001-2005
}

\author{
Hans Silfverberg
}

\begin{abstract}
Silfverberg, H. 2007: Changes in the list of Finnish insects during 2001-2005. Entomol. Fennica 18: 82-101.

During the period 2001-2005, 769 species have been added to the list of Finnish insects, and 213 species have been deleted from it. At the end of year 2005, a total of 20,533 insect species were known from Finland. The Finnish insect fauna is generally well known. Still, particularly for Diptera Nematocera and Hymenoptera Parasitica, and to some degree also in part of Hemiptera, there still remains much to be done.
\end{abstract}

H. Silfverberg, Zoological Museum, P.O.Box 26, 00014 Helsingfors, Finland. E-mail:hans.silfverberg@helsinki.fi

Received 10 March 2006, accepted 23 May 2006

\section{Introduction}

This paper is a sequel to a similar list published in 2001 (Silfverberg 2001, Entomol. Fennica 12: 227-243), which itself was a continuation of a series of lists published in 1921-1996 first by Hellén and then by Silfverberg. Insect species added to the fauna of Finland during the five-year period are listed, with references, as are those deleted from the fauna. A few previously unnoted changes have also been included.

For the new species the locality has generally been given, with its biogeographical province (for the provinces, see e.g. Heikinheimo \& Raatikainen 1981, Notulae Entomol. 61: 133-154). If the species has been reported from many localities, often only the provinces have been listed, or the abbreviation m.l. (= many localities) has been used. When Finland is mentioned as the locality, the original record gave no more exact location.

Some other abbreviations or signs must also be explained. Thus f.d. $=$ false determination and sp.dist. $=$ distinct species (previously considered a variety, a synonym or such like); in literature references $\mathrm{EF}=$ Entomologica Fennica nd $\mathrm{NE}=$ Notulae Entomologicae. A species enclosed in square brackets is considered accidentally im- ported, and has not been included in the species total. Again square brackets have been employed for the name used in the original record when different from the one used here. Other symbols are $*$ = new to science and $!=$ different from the check list mentioned for the order.

A thorough examination of earlier lists showed that a few species had been added twice. In order to correct the numbers, corresponding subtractions are made.

During the period covered by this list, 769 species have been added to the list of Finnish insects and 213 species have been deleted from it. At the end of year 2005, a total of 20,533 insect species were known from Finland.

\section{Additions and deletions from the list of Finnish insects}

\section{Collembola}

Deletions

Anurida brunsvigiensis Hüth. (= A. ellipsoides Stach). Fjellberg 1998 Fauna Entomol. Scand. 35:84.

Species accidentally counted twice 
Protaphorura fimata (Gisin). Silfverberg 1991 EF 2:9; Silfverberg 2001 EF 12:217.

Tullbergia arctica Wahlgr. Silfverberg 1991:9; Silfverberg 2001:217.

Mesaphorura hylophila Rusek. Silfverberg 1991:9; Silfverberg 2001:218.

- tenuisensillata Rusek. Silfverberg 1991:9; Silfverberg 2001:218.

Additions

Isotoma anglicana Lubb. Ta, Tb. Huhta \& al. 2005 Memoranda Soc. Fauna Flora Fenn. 81:62. Lepidocyrtus violaceus (Geoffr.) Tb. ibid.:62.

Present number: $218-5+2=215$ spp.

\section{Thysanura}

Additions

Ctenolepisma lineata (F.) N: Helsingfors. Huldén 2001 Sahlbergia 6:37.

[ - longicaudata Esch. N: Esbo \& Helsingfors. ibid.:37.]

Present number: $4+1=5 \mathrm{spp}$.

\section{Odonata}

Additions

Sympecma paedisca Brau. Ab: Dragsfjärd Örö. Kullberg 2004 Suomen Luonto 63(7):17.

Aeshna mixta Latr. Al, Ab, N. Kauppinen 2002 Suomen Luonto 61(10):16. Hildén \& al. 2003b Memoranda Soc. Fauna Flora Fennica 79:76. Hämäläinen, Huldén \& Karjalainen 2004 Sahlbergia 8:49.

Present number: $52+2=54$ spp.

\section{Psocoptera}

Additions

Psocus flavonimbatus Rost. Ok: Kuhmo. Lienhard \& Kanervo 2002 EF 13:59.

Mesopsocus helveticus Lienh. Ab, N. Kanervo 2002 Sahlbergia 7:37.

Present number: $65+2=67$ spp.

\section{Phthiraptera}

Deletions

Menacanthus vistulanus Eichl. \& Zlot. (= M. curru- cae ssp.) Hackman 1994 Memoranda Soc. Fauna Flora Fenn. 70:59.

Holomenopon nyrocae (Blagov.) (= H. leucoxanthum ssp.) ibid.:41.

Actornithophilus laveni Eichl. (= A. ochraceus ssp.) ibid.:46.

Ricinus bombycillae (Denny) (intermedius (Piag.)) (=R. elongatus ssp.) ibid.:56.

Craspedonirmus frontatus (Nitzsch) $(=$ C. colymbinus ssp.) ibid.:36.

Columbicola bacillus (Gieb.) (= C. columbae ssp.) ibid.:53.

Saemundssonia melanocephala (Burm.) (= S. sternae ssp.) ibid.:51.

Lunaceps phaeopi (Denny) (= L. numenii ssp.) ibid.:49.

Allobrueelia viscivora (Denny) (= A. marginata $\mathrm{ssp}$.) ibid.:57.

Docophorulus bischoffi Eichl. (=D. merulae ssp.) ibid.:57.

Cypseloecus microsomaticus (Tand.) (hirundinis (Piag.)) (=C. excisus ssp.) ibid.:55.

Pediculus capitis (DeG.) (= P. humanus L., var.) Leo \& Barker 2005 Parasitol. Res. 98:45.

Additions

Menacanthus merisuoi Eichl. Finland (on Nucifraga caryocatactes) Hackman 1994 Memoranda Soc. Fauna Flora Fenn. 70:62.

Lanicanthus camelinus inaequalis (Piag.) Finland (on Lanius collurio) ibid.:61.

Austromenopon vanelli Zlot. Finland (on Vanellus vanellus) ibid.:47.

Holomenopon clypeilargum Eichl. Finland (on Mergus merganser) ibid.:42.

Larithophilus piceus (Denny) Finland (on Larus argentatus) ibid.:51.

Laemobothrion maximum (Scop.) Finland (on Haliaeetus albicilla) ibid.:42.

Lagopoecus tetrastei Bech. Finland (on Tetrastes bonasia) ibid.:45.

Anaticola rheinwaldi Eichl. \& Vasj. Finland (on Branta bernicla) ibid.:39.

Columbicola claviformis (Denny) (sp. dist.) Finland (on Columba palumbus) ibid.:52.

Aquanirmus emersoni Edw. Finland (on Podiceps griseigena) ibid.:37.

Lunaceps falcinellus Timm. Finland (on Limicola falcinellus) ibid.:48.

Strigiphilus glaucidii Zlot. (sp. dist.) Finland (on Glaucidium passerinum) ibid.:53.

Picophilopterus arcticus Carr. Finland (on Picoides tridactylus) ibid.:55.

Docophorulus hansmanchi Eichl. \& Vasj. Finland (on Loxia leucoptera) ibid.:63.

Present number: $292-12+14=294$ spp. 


\section{Hemiptera}

Deletions

Aradus crassicornis Boh. (= A. betulinus Fall.). Heiss 2001 Catal. Heteropt. Pal. Reg. 4:17.

- pictus Bärenspr. (f.d., = A. obtectus Vásárh.). ibid $: 17$.

Additions

Calocoris affinis (H.-Sch.) N: Espoo, Helsinki. Albrecht \& al. 2004 Sahlbergia 8:65.

Phytocoris reuteri (Saund.) Ab: Turku, N: Hanko. ibid.:65.

Europiella artemisiae (Beck.) (obscura (J.Sahlb.)). Finland. Schuh, Lindskog \& Kerzhner 1995 Proc. Entomol. Soc. Washington 97:386.

Liorhyssus hyalinus (F.) Ab: Nagu. Ollikainen \& Rinne 2005 Sahlbergia 10:65.

Amphiareus obscuriceps (Popp.) Ka: Hamina. Albrecht \& al. 2004:66.

Odontoscelis fuliginosa (L.) $N$ : Pyhtää. ibid.:67

Dybowskyia reticulata (Dall.) Ka: Hamina. ibid.:67.

Eysarcoris aeneus (Scop.) Ka: Hamina. ibid.:67.

Cyphostethus tristriatus (F.) Ka: Vehkalahti. ibid.:67.

Aradus obtectus Vásárh. (A. pictus auct.). Finland. Heiss 2001 Catal. Heteropt. Pal. Reg. 4:16.

Kelisia praecox Haupt $A b, N$. Albrecht \& al. 2004:68.

Stenocranus fuscovittatus (Stål) $N$ : Pernå. ibid.:68.

Populicerus albicans (Kirschb.) N, Ka. ibid.:68.

Macropsidius sahlbergi (Flor) Sa: Lappeenranta. ibid.:68.

Macropsis scutellata (Boh.) Finland. Söderman 2004 Beitr. Zikadenk. 7:28.

Oncopsis avellanae Edw. Finland. ibid.:26.

Alebra albostriella (Fall.) Sa: Joutseno. Albrecht \& al. 2004:68.

- neglecta Wagn. Sa: Joutseno. ibid.:69.

- wahlbergi (Boh.) Ab, N. ibid.:69.

Vilbasteana oculata (Lindb.) N, Ka, Sa. ibid.:69.

Fagocyba carri (Edw.) Ab: Turku Ruissalo. ibid.:69.

Edwardsiana ampliata (Wagn.) N: Helsinki. ibid.:69.

- lanternae (Wagn.) N, Ta, Sa. ibid.:69.

- gratiosa (Boh.) N: Pyhtää, Ka: Vehkalahti. ibid.:69.

Eupterycyba jucunda (H.-Sch.) Ab: Mietoinen. ibid.:69.

Typhlocyba quercus (F.) Al, Ab, N, Ka, Ta, Sa, Kb. ibid.:69.

Zygina angusta Leth. Ab: Rymättylä. ibid.:70.

Balclutha arhenana (Dlab.) Al, Ab, N, Ka, Ta, Sa, Kb. ibid.: 70

- boica (Wagn.) N, Oa, Sb, Om, Ob. ibid.:70.

Scleroracus identicus Tish. Finland. Söderman 2004:26.
Laburrus impictifrons (Boh.) Sa: Lappeenranta. Albrecht \& al. 2004:71.

Metalimnus steini (Fieb.) Finland. Söderman 2004:28.

Paralimnus zachvatkini Em. Finland. ibid.:28.

Parapotes reticulatus (Horv.) N: Hangö. Albrecht \& al. 2004:71.

Calamotettix taeniatus (Horv.) $\mathrm{Al}, \mathrm{N}, \mathrm{Ka}, \mathrm{Sa}$. ibid.:71.

Mongolojassus bicuspidatus (J.Sahlb.) Sa: Lappeenranta. ibid.:71.

Aphalara crispicola Oss. $N$ : Helsinge, $S a$ : Mikkeli (O. Heikinheimo). Ossiannilsson 1992 Fauna Ent. Scand. 26:48.

Cacopsylla melanoneura (Först.) Ab, N, Ka, Ta. Albrecht \& al. 2004:71.

Trioza rotundata Flor St: Ikaalinen. Mattila 2002 Diamina 11:32.

Pemphigus populi Courch. Ab, N, Ka, Ta, Sa, Oa. Albrecht \& al. 2004:72.

Aphis erigerontis Holm. Ta: Nastola. ibid.:73.

- newtoni (Theob.) $N$ : Helsinki. ibid.:74.

- $\quad$ selini (Börn.) N: Helsinki, Pernå. ibid.:74.

- viburni Scop. N: Sibbo, Sa: Punkaharju. ibid.:74.

Brachycaudus tragopoginis (Kalt.) Ab, N. ibid.:75.

Coloradoa campestrella Oss. Ab: Pargas. ibid.:75.

Dysaphis centaureae (Börn.) Al: Hammarland. ibid.: 75 .

- newskyi (Börn.) Ta, Oa. Heie 1995 Fauna Ent. Scand. 31:175. Albrecht \& al. 2004:75

Hyperomyzus lampsanae Börn. N, Ta. Albrecht \& al. 2004:75.

Macrosiphoniella asteris (Walk.) Ab: Dragsfjärd. ibid.:75.

Pleotrichophorus duponti HRL Oa: Närpes. ibid.:75.

Titanosiphon artemisiae (Koch) Ab: Dragsfjärd. ibid.:75.

Tuberolachnus salignus (Gmel.) Ab, N, Ka, Ta, Sa. ibid.:76.

Accidentally omitted from check-list in 1984.

Typhlocyba bifasciata Boh. Silfverberg 1981 NE 61:47.

Present number: $1,376-2+54=1,428$ spp.

\section{Thysanoptera}

Additions

Lispothrips crassipes (Jabl.) Sb: Joroinen. Kettunen \& al. 2005 EF 16:249.

Hoplothrips carpathicus Pel. Sb: Jäppilä, Joroinen. ibid.:248.

Liophloeothrips hungaricus (Priesn.) Sb: Jäppilä. ibid.:249.

- glaber Priesn. Sb: Joroinen. ibid.:249.

Present number: $114+4=118$ spp. 


\section{Neuroptera}

Additions

Chrysopa commata Kis \& Ujh. (sp. dist.) Finland. Popov 2002 Acta Zool. Hungar. 48, Suppl. 2:284.

Present number: $62+1=63$ spp.

\section{Coleoptera}

Silfverberg, H. 2004a. Enumeratio nova Coleopterorum Fennoscandiae, Daniae et Baltiae. - Sahlbergia 9:1-111.

- 2004b. Enumeratio nova Coleopterorum: errata. — Sahlbergia 9:122.

Deletions

Haliplus wehnckei Gerh. (=H. sibiricus Motsch.). Lundmark \& al. 2001 Insect Syst. Evol. 32:248.

Patrobus australis J.Sahlb. (= P. septentrionis Dej.). Zamotajlov 2003 Acta Soc. Zool. Bohem. 67:241.

Helophorus pumilio Er. (f.d., = H. strigifrons Thoms.). Valtonen 2001 Sahlbergia 6:38.

- obscurus Muls. (f.d.). ibid.:38.

Quedius pseudoumbrinus Lohse (=Q. umbrinus Er.). Assing 1999 Entomol. Blätter 95:39.

Tytthaspis sedecimpunctata (L.) (collected outside Finland). Biström \& al. 2001 Sahlbergia 6:10, Huldén 2001 Sahlbergia 6:38.

Stenostola ferrea (Schr.) (f.d., = S. dubia (Laich.)). Clayhills 2003 Sahlbergia 7:100.

Phytoecia nigricornis (F.) (f.d., = P. cylindrica (L.), otherwise collected outside Finland). Heliövaara, Mannerkoski \& Siitonen 2004 Suomen sarvijäärät, p. 258.

Galerucella kerstensi Lohse (= G. sagittariae (Gyll.)). Silfverberg 2004a:82.

!Psylliodes isatidis Heik. (f.d., = P. crambicola Lohse). Wanntorp \& Ödegaard 2005 Entomol. Tidskr. 126:193.

Miarus fennicus Kang. (= M. campanulae (L.)). Silfverberg 2004a:93

Additions

!Badister sodalis (Duft.) N: Helsinki. Perkiömäki \& Rutanen 2005 Sahlbergia 10:28.

Harpalus rufipalpis Sturm Ab: Dragsfjärd Örö. Rassi 2004 Sahlbergia 8:100.

Microderes brachypus (Stev.) Finland (S. Karjalainen). Silfverberg 2004a:14, 99.

Bradycellus csikii Laczó Finland (H. Lappalainen). ibid.: 14, 99 .

- verbasci (Duft.) Finland (P. Rassi). ibid.:14, 99.
Hydrochus elongatus (Schall.) Finland (S. Karjalainen). ibid.:15, 99.

Berosus signaticollis (Charp.) Al: Föglö. Rassi 2001 Sahlbergia 6:37.

Margarinotus terricola (Germ.) Finland (P. Martikainen). Silfverberg 2004a:18, 99.

Acrotrichis lucidula Rossk. Ta: Somero. Sörensson \& Rutanen 2003 Sahlbergia 7:96.

- sjobergi Sundt Ok: Kuhmo. ibid.:98.

Tachinus schneideri Luze Finland (I. Rutanen). Silfverberg 2004a:28, 99.

Aleochara haematoptera Kr. Finland (T. Clayhills). ibid.:29, 99.

Aloconota strandi Ben. Ab: Suomusjärvi. Iivarinen 2001 Sahlbergia 6:37.

Philhygra pseudoelongatula (Bernh.) Finland (P. Rassi). Silfverberg 2004a:32, 99.

Atheta indubia (Sharp) Finland (J. Muona). ibid.:33, 99.

Trichiusa immigrata Lohse $A b$ : Nummi-Pusula (I.Mannerkoski); N: Hyvinkää (I. Rutanen). Mannerkoski 2001 Sahlbergia 6:37. Hildén \& al. 2003a Memoranda Soc. Fauna Flora Fenn. 78:68.

Bolitochara obliqua Er. Finland (M. Landvik). Silfverberg 2004a:36, 99.

Gabronthus sulcifrons (Sharp) Ab, N, Ta, Kb, Om, Lk. Smetana \& Clayhills 2004 Entomol. Tidskr. 125:121. Silfverberg 2004:43, 99.

Philonthus alpinus Epp. Finland (P. Rassi). Silfverberg 2004a:44, 99.

Quedius vexans Epp. Finland (P. Rassi). ibid.:45, 99.

* Trixagus leseigneuri Muona N: Helsingfors Drumsö (S. Stockmann). Muona 2002 Bull. Soc. Entomol. France 107:187.

Negastrius arenicola (Boh.) Li: Utsjoki. Gillerfors 2003 Entomol. Tidskr. 124:175.

[Dermestes haemorrhoidalis Küst. Ab: Naantali. Clayhills 2001 Sahlbergia 6:37.]

[Attagenus trifasciatus (F.) Finland (J. Muona). Silfverberg 2004a:56, 99.]

[Dinoderus bifoveolatus Woll. $N$ : Espoo (Valovirta). ibid.:63.]

[Lyctus hipposideros Lesne $N$ : Helsingfors. Geis 2002 Mitt. Internat. Entomol. Ver., Suppl. 10:43.]

[Trogoxylon praeustum (Er.) N: Helsingfors. ibid.:52.]

Dorcatoma flavicornis (F.) Finland (J. Mattila, P. Ahlroth \& M. v.Numers). Silfverberg 2004a:58, 99.

Meligethes subaeneus Sturm N. Audisio \& al. 2001 Europ. Journ. Entomol. 98:93.

Cryptarcha undata (Ol.) Finland (J. Mattila \& P. Ahlroth). Silfverberg 2004a:62, 99.

Monotoma quadricollis Aubé $\mathrm{Al}, \mathrm{Ab}, \mathrm{N}, \mathrm{Ka}, \mathrm{Ta}, \mathrm{Sa}$, $T b$. Rutanen 2003 Sahlbergia 8:25.

Cryptolestes weisei (Rtt.) Finland (I. Rutanen). Silfverberg 2004a:63, 99. 
Atomaria pseudatra Rtt. Finland (I. Rutanen). ibid.:64, 99.

Calvia decemguttata (L.) Finland (S. Karjalainen). ibid.:67, 99.

Dienerella ruficollis (Marsh.) Finland (I. Rutanen). ibid.:68, 99.

Corticarina truncatella (Mannh.) Finland (P. Martikainen). ibid.:69, 99.

Stricticomus tobias (Mars.) Finland (S. Karjalainen). ibid.: 75, 99.

[Plagionotus detritus (L.) N: Esbo, Helsingfors. Biström 2004a Sahlbergia 9:127.]

Stenostola dubia (Laich.) (nec ferrea (Schr.)). Finland. Clayhills 2003 Sahlbergia 7:100.

Otiorhynchus lugdunensis Boh. Finland (I. Mannerkoski). Silfverberg 2004a:99.

Ceutorhynchus arquatus (Hbst.) Finland (P. Rassi). ibid.:96, 99.

Carphoborus cholodkowskyi Spess. Ta: Korpilahti. Hyvärinen \& Mattila 2004 Sahlbergia 8:82.

Species accidentally omitted for Finland

in check list.

!Veraphis engelmarki Franz Finland. Silfverberg 2004b:122.

!Tachyporus quadriscopulatus Pand. Finland. ibid.: 122 .

!Rhagonycha fulva (Scop.) Finland. ibid.:122.

!Cryptophagus obsoletus Rtt. Finland. ibid.:122.

!Notaris bimaculatus (F.) Finland. ibid.:122.

Present number: $3,632-11+36=3,657 \mathrm{spp}$.

\section{Trichoptera}

Deletions

Apatania stylata Navás (f.d.) Salokannel \& al. 2004 Sahlbergia 9:119.

Additions

Apatania forsslundi Tob. Lk: Muonio, Li: Inari. Mattila \& Salokannel 2004 Diamina 13:4. Salokannel \& al. 2004 Sahlbergia 9:120.

- $\quad$ muliebris McLachl. Le: Saana. Ilmonen \& Rinne 2005 Diamina 14:4.

Grammotaulius nitidus (Müll.) N: Tammisaari, Harparskog. Salokannel 2003 Diamina 12:2. Salokannel 2004 Sahlbergia 8:100. Salokannel \& al. 2004:120.

Present number: $213-1+3=215$ spp.

\section{Lepidoptera}

Kullberg, J., Albrecht, A., Kaila, L. \& Varis, V. 2002. Checklist of Finnish Lepidoptera - Suomen perhosten luettelo. - Sahlbergia 6:45-190.

Deletions

Elachista regificella Sirc. (f.d., = E. tengstromi Kaila \& al.). Kaila \& al. 2001 EF 12:157.

Clepsis consimilana (Hübn.) (f.d., = C. pallidana (F.)) Kullberg \& al. 2002:100. Mutanen \& al. 2003 Baptria 28(2):15.

Eucosma jaceana (H.-S.) (f.d., = E. flavispecula Kuzn.). Kullberg \& Sundell 2002 Baptria 27:75.

!Melanargia galathea (L.) dubious record. Nupponen \& al. 2002 Baptria 27:130.

Selidosema plumarium (D. \& Sch.) (f.d., = S. brunnearium (Vill.)) Kullberg \& al. 2002:155.

Diarsia florida (Schm.) (=D. rubi (View.)) Kullberg \& al. 2002:159.

Additions

!Stigmella aceris (Frey) Al: Eckerö. Mutanen, Itämies, Junnilainen, Kaitila, Kullberg, Mutanen \& Välimäki 2003 Baptria 28(2):6.

Tinea pallescentella Haw. N: Kullberg \& al. 2002:57. Mutanen \& al. 2003:6.

Caloptilia robustella Jäckh $N$ : Espoo (M. Tyni); Inkoo (T. Ranki \& J. Junnilainen). Kullberg \& al. 2002:60. Hildén \& al. 2002 Memoranda Soc. Fauna Flora Fenn. 77:20. Mutanen \& al. 2003:6. Mutanen \& Nupponen 2003 Baptria 28(4):7.

!Leucospilapteryx omissella (Staint.) $\mathrm{Ab}, \mathrm{N}, \mathrm{Ka}, \mathrm{Kb}$. Mutanen, Nupponen \& Välimäki 2005 Baptria 30:62. Mutanen, Nupponen \& Kaitila 2005 Baptria $30: 131$.

Phyllonorycter roboris (Zell.) N: Espoo (M. Tyni). Kullberg \& al. 2002:60. Hildén \& al. 2002:20. Mutanen \& al. 2003:7.

- heringiellus (Grönl.) (sp. dist.) $A l, A b, N, K a, T a$, $\mathrm{Sa}, \mathrm{Om}, \mathrm{Ob}$. Kullberg \& al. 2002:61. Mutanen, Nupponen \& Välimäki 2005:66.

- maestingellus (Müll.) N: Espoo (K. Nupponen). Kullberg \& al. 2002:60. Mutanen \& Nupponen 2003:7.

! - issikii (Kum.) N, Ta, Kl. Mutanen, Nupponen \& Välimäki 2005: 62.

Leucoptera orobi (Staint.) (sp. dist.) $\mathrm{Al}, \mathrm{Ka}, \mathrm{Ta}, \mathrm{Sa}$ Kullberg \& al. 2002:65.

!Exaeretia praeustella (Reb.) $N$ : Tammisaari Jussarö (T. \& K. Nupponen). Mutanen, Nupponen \& Kaitila 2005:124.

!Ethmia dodecea (Haw.) N: Tammisaari Hästö-Busö. ibid.: 125 .

Agonopterix purpurea (Haw.) Ab: Dragsfjärd (K. Helomaa \& K. Saloranta). Kullberg \& al. 2002:66. 
Kullberg 2002a Tvärminne Stud. 9:61. Mutanen \& Nupponen 2003:7.

- multiplicella (Ersch.) Ka: Virolahti. Kullberg \& al. 2002:66. Mutanen \& Nupponen 2003:7.

Depressaria albipunctella (D. \& Sch.) Ab: Dragsfjärd Örö (A. \& J. Kullberg) Kullberg \& al. 2002:66. Kullberg 2002a:61. Kullberg 2002b Baptria 27:77.

* Elachista tengstromi Kaila \& al. (regificella auct.) Al-Tb. Kaila \& al. 2001 EF 12:164.

- abiskoella Bengts. Li: Inari (E. \& L. Laasonen) Kullberg \& al. 2002:68. Laasonen \& Laasonen 2003 Baptria 28:23. Mutanen \& Nupponen 2003:8.

Metriotes lutarea (Haw.) N: Vantaa (J. Kaitila). Kullberg \& al. 2002:71. Mutanen \& al. 2003:7.

Coleophora lutipennella (Zell.) $N$ : Inkoo (J. Kaitila). Kullberg \& al. 2002:71. Mutanen \& al. 2003:8.

- follicularis (Vall.) Ka: Vehkalahti (M. Tyni). Kullberg \& Tyni 2002 Baptria 27:19. Kullberg \& al. 2002:74.

!* - jaernaensis Björkl. \& Palmqv. Ab: Piikkiö (B. Wikström), Ta. Björklund \& Palmqvist 2002 Ent. Tidskr. 123:99.

! - conyzae Zell. N: Tammisaari (T. \& K. Nupponen). Mutanen, Nupponen \& Välimäki 2005:63.

!Pancalia leuwenhoekella (L.) $N$ : Ruotsinpyhtää (J. Junnilainen). Mutanen \& Nupponen 2003:8.

Carpatolechia decorella (Haw.) Al: Lemland (J. Junnilainen). Kullberg \& al. 2002:77. Mutanen \& al. 2003:8.

!Cosmopterix scribaiella Zell. $N$ : Tammisaari, Inkoo. Mutanen, Nupponen \& Kaitila 2005:126.

!Chionodes tragicellus (Heyd.) N: Helsinki (M. Tyni). Mutanen, Nupponen \& Välimäki 2005:63.

!Scrobipalpa bryophiloides Povol. Ka: Kotka (L. Luukkonen \& R. Teriaho); Virolahti (K. Saloranta \& K. Vaalamo). Mutanen \& Nupponen 2003:8.

!Cosmardia moritzella (Treit.) Ab: Dragsfjärd Örö. Kullberg 2004 Baptria 29:90. Mutanen, Nupponen \& Kaitila 2005:127.

Caryocolum blandulellum (Tutt) Ab: Dragsfjärd Örö (A. \& J. Kullberg). Kullberg \& al. 2002:79. Kullberg 2002a:61. Kullberg 2002b:79.

Zygaena minos (D. \& Sch.) Ab: Turku (O. Ermala). Kullberg \& al. 2002:81.

!Zeuzera pyrina (L.) Al: Lemland (K. Vaalamo \& B. Wikström). Mutanen, Nupponen \& Kaitila 2005:126.

*Aethes kyrkii Itäm. \& Mut. Ob, Ks, Lk. Kullberg \& al. 2002:84 [Aethes sp. nr. cnicana]. Itämies, Mutanen \& Lankinen 2003 Ins. Syst. Evol. 34:8.

!Cochylis atricapitana (Steph.) Ab: Dragsfjärd Örö. Kullberg 2003b Baptria 28(4):21.

!Bactra suedana Bengtss. Al: Kökar. Mutanen, Nupponen \& Välimäki 2005:63.

Endothenia pullana (Haw.) N: Pyhtää. Kullberg \& al. 2002:87. Mutanen \& al. 2003:8.
! - gentianacea (Hübn.) Kb: Ilomantsi (J.-P. Kaitila \& E. Tuomisto). Mutanen, Nupponen \& Kaitila 2005:127.

!Ancylis rhenana Müll.-R. Ab, N, Ka. Laasonen \& Laasonen 2005 Baptria 29:122. Mutanen, Nupponen \& Kaitila 2005:127.

Pelochrista mollitana (Zell.) N: Hanko (J.-P. Kaitila); $N$ : Tammisaari (K. \& T. Nupponen). Kullberg \& al. 2002:90. Mutanen \& Nupponen 2003:9.

Eucosma flavispecula Kuzn. Ab, N, Ka. Kullberg \& Sundell 2002 Baptria 27:76. Hildén \& al. 2003a Memoranda Soc. Fauna Flora Fenn. 78:68. Mutanen \& Nupponen 2003:12. Mutanen, Nupponen \& Välimäki 2005:67.

! - fulvana Steph. Al, Ab, N, Ka, Ta, Kl. Mutanen, Nupponen \& Kaitila 2005:128.

Epiblema cnicicolanum (Zell.) $N$ : Hanko Russarö (J. Kullberg). Kullberg \& al. 2002:91. Kullberg 2002a:61.

Rhyacionia logaea Durr. (sp. dist.) $A l, A b, N, K a, S t$, Ta, Sa. Kullberg \& al. 2002:92.

!Cydia microgrammana (Guen.) N: Hanko (P. Sundell). Mutanen, Nupponen \& Välimäki 2005:64.

! - lobarzewskii (Now.) Al: Maarianhamina (K. Vaalamo \& B. Wikström). Mutanen, Nupponen \& Kaitila 2005:128.

! - oxytropidis (Mart.) Ka: Virolahti (J. \& P. Kaitila \& S. Korpela). ibid.:128.

!Grapholita janthiniana (Dup.) N: Hanko (T. Mutanen \& T. Ojala). Mutanen, Nupponen \& Välimäki 2005:64.

Pammene luculentana (Kuzn.) Ta: Orivesi, Kl: Parikkala. Laasonen \& Laasonen 2002 Baptria 27:29. Kullberg \& al. 2002:93. Mutanen \& al. 2003:8.

Tebenna pretiosana (Dup.) $N$ (E. Franssila), Ka (J. \& P. Kaitila). Kullberg \& al. 2002:94. Mutanen, Nupponen \& Kaitila 2005:131.

Stenoptilia zophodactyla (Dup.) Ab: Dragsfjärd Örö (A. \& J. Kullberg) Kullberg \& al. 2002:95. Kullberg 2002a:61. Kullberg 2002b:80.

!Oidaematophorus vafradactylus Svenss. N: Tammisaari Jussarö (T. \& K. Nupponen). Mutanen, Nupponen \& Kaitila 2005:128.

!Salebriopsis albicilla (H.-S.) Ab: Dragsfjärd Örö. Kullberg 2003b:22.

Etiella zinckenella (Tr.) Ab: Dragsfjärd (K. Helomaa \& K. Saloranta). Kullberg \& al. 2002:97. Kullberg 2002a:61. Mutanen \& Nupponen 2003:9.

Nephopterix angustella (Hübn.) Ab: Dragsfjärd Örö (J. Kullberg). Kullberg \& al. 2002:98. Kullberg 2002a:61. Kullberg 2002b:81.

!Eurhodope cirrigerella (Zinck.) Kb: Kitee (M. Liljeblad \& J. Kanerva). Mutanen, Nupponen \& Välimäki 2005:64.

!Scoparia basistrigalis Knaggs $N$ : Hanko \& Tammisaari. Mutanen, Nupponen \& Välimäki 2005:64.

Catoptria verella (Zinck.) Al, Ab, N, Ka. Kullberg \& al. 2002:100. Kullberg 2002a:61. Kullberg \& 
Junnilainen 2002 Baptria 27:86.

Pediasia luteella (D. \& Sch.) N, Ka: Virolahti (J.-P. Kaitila). Kaitila \& Nupponen 2002 Baptria 27:19. Kullberg \& al. 2002:100.

Evergestis limbata (L.) Al: Kökar, $N$ : Espoo. Kullberg \& al. 2002:102. Mutanen \& Nupponen 2003:9. Mutanen, Nupponen \& Välimäki 2005:67.

!Leptidea reali Reiss. Al, N. Saarinen 2005 Baptria 29:114.

!Pontia callidice (Hübn.) Li: Utsjoki (T. Nupponen). Saarinen 2005:114. Mikkola 2005 Baptria 29 [recte 30]:101.

Apatura ilia (D. \& Sch.) N (B.-G. Kumlander, H. Takanen). Kullberg \& al. 2002:110. Saarinen 2003 Baptria 28:8.

!Limenitis camilla (L.) Ta: Valkeakoski. Nieminen \& Nieminen 2004 Diamina 13:2.

Sabra harpagula (Esp.) N: Inkoo (T. Ranki). Kullberg \& al. 2002:112.

Scopula caricaria (Reutti) N: Kirkkonummi (J. Junnilainen). Kullberg \& al. 2002:113.

Idaea ochrata (Scop.) Ab: Dragsfjärd Örö (A. \& J. Kullberg). Kullberg \& al. 2002:113. Kullberg 2002a:61. Mikkola 2002 Baptria 27:59. Kullberg 2003a Baptria 28(4):20.

!Rhodometra sacraria (L.) Ta: Hattula. Mikkola 2004 Baptria 29:26.

Eupithecia fraxinata Crewe (sp. dist.) $A l, A b, N$, St, $O a, O b$. Kullberg \& al. 2002:119.

Ligdia adustata (D. \& Sch.) N: Vantaa (J. Tamminen). Kullberg \& al. 2002:120. Kullberg \& Wettenhovi 2002 Baptria 27:99.

!Alsophila aescularia (D. \& Sch.) Sb: Suonenjoki. Leinonen, Ylönen \& Ylönen 2005 Baptria 30:71.

!Alcis bastelbergeri (Hirschke) Ka: Virolahti (L. Jalonen, O. Helminen \& I. Seuranen). Kaitila \& Jalonen 2004 Baptria 29:69.

!Peridea anceps (Gze.) N: Nurmijärvi (K. Keinänen). Hildén \& al. 2003b Memoranda Soc. Fauna Flora Fenn. 79:77.

Arctornis l-nigrum (Müll.) Ka: Virolahti (J.-P. Kaitila \& S. Korpela). Kullberg \& al. 2002:125. Mikkola 2002:59.

!Pelosia obtusa (H.-S.) Al: Kökar. Sundell \& Kaitila 2003 Baptria 28(4):16.

[Holomelina aurantiaca (Hb.) Sa: Ristiina. Sihvonen \& Sihvonen 2001 Baptria 26:121.]

!Phragmatobia luctifera (D. \& Sch.) N: Kirkkonummi. Helomaa 2005 Baptria 29 [recte 30]:112.

Schranckia intermedialis Reid Ka: Virolahti (M. \& T. Mutanen \& P. Välimäki). Kullberg \& al. 2002:128. Mikkola 2002:59.

- taenialis (Hübn.) Ka: Kotka (J. Suomi). Kullberg \& al. 2002:128. Mikkola 2002:59.

Calyptra thalictri (Borkh.) Kl: Simpele (M. Sinervirta). Kullberg \& al. 2002:129.

!Diachrysia zosimi (Hb.) Ka: Virolahti (R. Elfving).
Mikkola 2003 Helsingin Sanomat 27.7.:D10.

Acronicta cinerea (Hufn.) (sp. dist.) Ab-Le. Kullberg $\&$ al. 2002:131.

Eucarta virgo (Tr.) N: Hangö (A. Graeffe). Kullberg $\&$ al. 2002:131.

Aedia funesta (Hb.) Ab: Dragsfjärd Örö. Kullberg 2004:90.

Cucullia lychnitis Ramb. Ab: Kisko (K. Wettenhovi). Kullberg \& al. 2002:132.

! - chamomillae (D. \& Sch.) N: Hanko. Lehto 2004 Baptria 29:36.

Platyperigea albina (Ev.) $N, \mathrm{Ka}, \mathrm{Sa}$. Kullberg \& al. 2002:133. Mikkola 2002:59.

Oligia versicolor (Borkh.) N: Tammisaari (K. \& T. Nupponen). Kullberg \& al. 2002:136.

!Mesapamea remmi Rezb.-R. Al: Kökar. Lundsten \& Sihvonen 2003 Baptria 28(4):18.

Hadena bicruris (Hufn.) Al: Lemland (K. Vaalamo). Kullberg \& al. 2002:138.

Mythimna vitellina (Hübn.) $N, \mathrm{Ka}$. Kullberg \& al. 2002:139.

!Euxoa temera (Hübn.) N: Kirkkonummi (D. Lindblom). Peltokangas 2002 Baptria 27:50. Kullberg 2005 Baptria 30:36

! - vitta (Esp.) Ab, N. Kullberg 2005:36.

Present number: $2,426-6+89=2,509$ spp.

\section{Diptera}

Kahanpää, J. \& Winqvist, K. 2005. Check-list of Finnish flies: families Xylophagidae - Microphoridae. - Sahlbergia 10:10-27.

Deletions

Prionocera lapponica Tjed. (= P. recta Tjed.). Oosterbroek \& Theowald 1992 Catal. Pal. Dipt. 1:85.

Neolimnomyia leucophaea (Meig.) $(=N$. nemoralis (Meig.)). Savchenko, Oosterbroek \& Starý 1992 Catal. Pal. Dipt. 1:225.

Dicranomyia schineriana (Alex.) $(=D$. schineri Lacks.). ibid.:344.

Cricotopus alpestris Goetgh. $(=$ C. annulator Goetgh.). Ashe \& Cranston 1990 Catal. Pal. Dipt. 2:172.

Psectrocladius edwardsi Brund. (= P. limbatellus (Holmgr.). ibid.:218.

Tanytarsus decipiens Lindeb. (= T. lestagei Goetgh.). Ekrem 2004 Deutsche Entomol. Zschr. 51:108.

- palmeni Lindeb. (= T. lestagei Goetgh.). ibid.:108.

- socialis Lindeb. (= T. telmaticus Lindeb.). ibid.:113.

- simulans Lindeb. (= T. telmaticus Lindeb.). ibid.:113.

Bolitophila tarsata (May.) (= B. modesta Lacks.). 
Kallweit \& Plassmann 1999 Stud. Dipterol., Suppl. 2:17.

Brevicornu boreale (Lundstr.) $(=$ B. griseolum (Zett.)). Kjaerandsen 2005 Zootaxa 856:2.

Mycetophila tarsata Winn. ( $=$ M. occultans Lundstr.). Zaitzev 2003 Dipterol. Res. 14:268.

Phronia minuta Landr. (= P. longelamellata Strobl, nec auct.). Chandler 2001 Brit. Journ. Entomol. Nat. Hist. 13:241.

- appropinquata Strobl (= Trichonta vulcani (Dziedz.)). Zaitzev 2003:356.

Trichonta adunca Edw. (= T. atricauda (Zett.)). ibid.:339.

- nigricauda Lundstr. (= T. vulgaris Loew). ibid.:358.

Trichosia elegans (Winn.) (= Leptosciarella scutellata (Staeg.). Menzel \& Mohrig 1999 Stud. Dipterol., Suppl. 6:361.

- $\operatorname{coarctata}($ Winn.) (= Leptosciarella trochanterata (Zett.)). ibid.:358.

Plastosciara brachialis (Winn.) (= Cratyna nobilis (Winn.)). ibid.:280.

- brevicornis Tuomik. (= Cratyna perplexa (Winn.)). ibid.:284.

Camptochaeta uncinata Hippa \& Vilk. (= Corynoptera parvula (Winn.)). ibid.:242.

Lycoriella solani (Winn.) (= L. ingenua (Duft.)). ibid.:394.

- brevipila Tuomik. (= L. ingenua (Duft.)). ibid.:394.

Bradysia betuleti (Leng.) (= B. hilaris (Winn.)). ibid.:167.

- atroparva Frey (=B. nitidicollis (Meig.)). ibid.: 180 .

- degenerans Frey (= Scaptosciara tricuspidata (Winn.)). ibid.:501.

- myrmecophila Frey (= Scaptosciara curviforceps (Buk. \& Leng.)). ibid.:497.

Leptomorphus quadrimaculatus (Matsum.) (f.d., $=L$. subforcipatus Zaitz. \& Ševč.). Zaitzev \& Ševčik 2002 Acta Zool. Acad. Sci. Hungar. 48:203.

Sargus splendens Meig. (f.d., = S. flavipes Meig.). Kahanpää \& Winqvist 2005:20.

Odontomyia hydroleon (L.) (doubtful record). ibid.:13

Haematopota italica Meig. (f.d., = H. pluvialis (L.)). ibid.:13.

Cyrtopogon maculipennis (Macq.) (=C. flavimanus (Meig.)). Lehr 1988 Catal. Pal. Dipt. 5:237.

Cliorismia ardea (F.) (recorded outside Finland). Kahanpää \& Winqvist 2005:14.

- rustica (Panz.) (recorded outside Finland). ibid.:14.

Thereva circumscripta Loew (f.d., $=$ T. valida Loew). ibid.:14.

Bombylius albibarbis Zett. (= B. minor L.). ibid.:22. Anastoechus nitidulus (F.) (doubtful record). ibid.:14. Villa circumdata (Meig.) (f.d.). ibid.:14.
- halteralis (Kow.) (doubtful record). ibid.:14.

- paniscus (Rossi) (f.d., = V. modesta (Meig.)). ibid.:14.

Microphorella praecox (Loew) (doubtful record). ibid.:17.

Iteaphila furcata (Zett.) (doubtful record). ibid.:16.

Hilara flavipes Meig. (f.d.). Chvála 2005 Fauna Ent. Scand. 40:48.

- quadrifaria Strobl (f.d., =H. coracina Old.). Chvála 2002a EF 13:74.

- hirtella Coll. (f.d., = H. hirta Strobl). Chvála 2002b Acta Univ. Carol., Biol. 46:245. Chvála 2005:115.

- pseudochorica Strobl (f.d.). Kahanpää \& Winqvist 2005:15.

- quadrivittata Meig. (f.d., = quadrifasciata Chvála). ibid.:23.

- scrobiculata Loew (f.d.). ibid.:16.

- tetragramma Loew (doubtful record). ibid.:16.

Empis punctata Meig. (recorded outside Finland). ibid.: 15 .

Rhamphomyia tibialis Meig. (f.d.). ibid.:16.

- anomala Old. (f.d., = R. anomalipennis Meig.). ibid.:16.

- tephraea Meig. (f.d., = R. maculipennis Zett.). ibid.:16.

- aperta Zett. (=R. griseola Zett. (?)). ibid.:24.

- longestylata Frey (=R. caudata (Zett.)). ibid.:16.

- dentipes Zett. (= R. pilifer Meig.). ibid.:24.

- intermedia Frey (= R. pilifer Meig.). ibid.:24.

Chelifera astigma Coll. (doubtful record). ibid.:16.

Clinocera nigra Meig. (doubtful record). ibid.:15.

Medetera thunebergi Negr. (= M. excellens Frey). Grichanov 2002 Ent. Tidskr. 123:124.

Megaselia setifer (Lundb.) (= M. tumida (Wood)). Disney 2002 Bonn. zool. Beitr. 50:297. Listed with question mark by Hackman (1980).

Baccha obscuripennis (Meig.) (= B. elongata (F.)). Haarto \& Kerppola 2004 Sahlbergia 9:154.

Chrysotoxum elegans Loew (f.d., = C. octomaculatum Curt.). ibid.:154.

Platycheirus complicatus Beck. (f.d., = P. kittilaensis Dušek \& Laska). ibid.:154.

Pipizella brevis Luc. (f.d., = P. curta Viol.). ibid.:155.

- virens (F.) (f.d., = P. viduata (L.)). ibid.:155.

Cheilosia gracilis Hell. (= C. gigantea (Zett.)). ibid.:154.

- rotundicornis Hell. (= C. vernalis (Fall.)). ibid.: 154 .

- ruralis (Meig.) (=C. mutabilis (Fall.)). ibid.:154.

- $\operatorname{soror}($ Zett.) $($ f.d., $=$ C. longula (Zett.)). ibid.:154.

Chrysogaster viduata (L.) (f.d., = Melanogaster aerosa (Loew)). ibid.:154.

Orthonevra brevicornis (Loew) (f.d., = O. plumbago (Loew). ibid.:154.

Eristalis vitripennis $\mathrm{Strobl}$ (= E. rupium F.). Hippa, Nielsen \& Steenis 2001 Norw. J. Entomol. $48: 312$ 
Temnostoma bombylans (F.) (f.d., = T. angustistriatum Kriv.). Krivosheina \& Ståhls 2004 Sahlbergia 8:97.

Eudorylas fascipes (Zett.) (f.d., = E. coloratus (Beck.)). Kehlmaier 2005 Verh. Naturwiss. Ver. Hamburg 41:223.

Psila buccata Fall. (= Chamaepsila gracilis (Meig.)). Soós 1984 Catal. Pal. Dipt. 9:30.

Homalocephala bipunctata (Loew) $(=H$. albitarsis (Zett.)). Zaitzev 1984 Catal. Pal. Dipt. 9:65.

Campiglossa borealis Port. (= C. grandinata (Rond.)). Foote 1984 Catal. Pal. Dipt. 9:74.

Sapromyza imitatrix Czerny (=S. opaca Beck.). Papp 1984 Catal. Pal. Dipt. 9:214.

Palloptera arcuata (F.) (f.d., = P. trimacula Meig.). Morge 1984 Catal. Pal. Dipt. 9:246.

Lonchaea laticornis Meig. (f.d., = L. caledonica McGow. \& Roth.). MacGowan \& Rotheray 2000 Dipter. Dig. 7:39.

Anthomyza sordidella (Zett.) (=A. gracilis Fall.). Andersson 1984 Catal. Pal. Dipt. 10:52.

Geomyza consobrina Zett. (= G. apicalis Meig.). Soós 1984 Catal. Pal. Dipt. 10:55.

Ischiolepta paracrenata (Duda) (=I. denticulata (Meig.)). Papp 1988 Catal. Pal. Dipt. 10:70.

Limosina antennata Duda (= Pullimosina moesta Villen.). Rohaček 2000 Bull. Soc. Ent. France 105:474.

Chlorops zonulatus Wahlgr. (f.d., = C. kirigaminensis Kanm.). Nartshuk 2002 Dipterol. Res. 13:16.

Orygma luctuosum Meig. (collected outside Finland). Pont \& Meier 2002 Fauna Ent. Scand. 37:38.

Cordilura melanacra (Loew) (= Scoliaphleps ustulata (Zett.). Šifner 2003 Acta Mus. Natn. Prag., Ser. B 59:19.

Cosmetopus fulvipes (Zett.) (=C. dentimanus (Zett.)). Gorodkov 1986 Catal. Pal. Dipt. 11:23.

Parallelomma paridis Her. (= Americina vittata (Meig.). Šifner 2003:65.

Fannia glaucescens (Zett.) (=F. armata (Meig.)). Pont 2002 Ins. Syst. Evol. 33:107.

Hydrotaea occulta (Meig.) (=H. armipes (Fall.)). Pont 1986 Catal. Pal. Dipt. 11:75.

Hebecnema affinis Mall. (=H. vespertina (Fall.)). ibid.:162.

Spilogona fumipennis (Zett.) (=S. contractifrons (Zett.)). ibid.:166.

Sarcophaga schulzi Müll. (= S. carnaria (L.)). Pape 1987 Fauna Ent. Scand. 19:176.

Species accidentally counted twice

Hydrobaenus pilipes (Mall.) Hackman 1980 NE 60:27; Silfverberg 1981 NE 61:53.

Propsilocerus [Tokunagayusurika] jacuticus (Zvet.) Silfverberg 1991 EF 2:14; Silfverberg 1996 EF $7: 45$.
Chironomus macani Freem. Hackman 1980:27; Silfverberg 2001 EF 12:232.

- melanotus Keyl Silfverberg 1981:53; Silfverberg 2001:232.

Pachyneura fasciata Zett. Hackman 1980:29; Silfverberg 1996:45.

Eupeodes [Metasyrphus] abiskoensis (Dušek \& Las.) Silfverberg 1996:45; Silfverberg 2001:234.

Admontia [Trichopareia] grandicornis (Zett.) Hackman 1980:141; Silfverberg 1986:141.

Prosopea nigricans (Egg.) Silfverberg 1981:55; Silfverberg 1986:142.

Species reported and later deleted

Sciophila hebes Johans. (= S. antiqua Chandl.) Ok: Kuhmo. Komonen 2001 Sahlbergia 6:37. Polevoi \& Jakovlev 2004:138.

Simulium sublacustre Dav. Ks: Kuusamo. Kuusela 1992:42. Synonym of $S$. rostratum (Lundstr.) Yankovsky 2002 Opred. Faune Rossii 170:22.

Additions

Tipula laccata Lundstr. \& Frey Ks: Kuusamo (H. Bruun). Viramo 1992 Oulanka Rep. 10:36.

- wahlgreni Lacksch. Sb: Savonranta. Starì \& Salmela 2004 EF 15:47.

Ula mixta Starì $A b, T a, S b$. Salmela 2001b EF 12:152. Starì \& Salmela 2004:47.

- kiushiuensis Alex. $N$ : Espoo, Kb: Ilomantsi, $K s$ : Kuusamo. Salmela \& Piirainen 2003 Norw. Journ. Entomol. 50:76.

Dicranota gracilipes Wahlgr. Li: Inari. Salmela 2001a Diamina 10:21.

- pavida (Halid.) Ab, St, Ta. Salmela 2001b:152.

Paradelphomyia fuscula (Loew) Ab, N, Ta. Krogerus 1936 Notulae Entomol. 16:89. Salmela 2001b:150. Accidentally omitted from check list in 1980.

Adelphomyia punctum (Meig.) Ta: Urjala. Salmela \& Härmä 2004 Sahlbergia 9:141.

Erioptera pederi Tjed. Ta: Somero. Salmela 2001 b: 150.

Molophilus bifidus Goetgh. Ab: Kiikala. ibid.:150.

- bihamatus Meij. Ta: Ruovesi. ibid.:150.

- corniger Meij. Ab, N, Ta. ibid.:151.

Gnophomyia acheron Alex. Sb: Savonranta. Starì \& Salmela 2004:47.

Lipsothrix ecucullata Edw. Ab, N, Ta, Sb. Salmela 2001b:151. Starì \& Salmela 2004:47.

Elephantomyia krivosheinae Savch. Sb: Savonranta. $K b$ : Ilomantsi. Polevoi 2001b Metsähall. luonnonsuoj.julk. A 131:191. Starì \& Salmela 2004:47.

Helius flavus (Walker) Ta: Tampere. Salmela 2002 Diamina 11:32. 
Dicranomyia occidua Edw. Ks: Kuusamo (H. Bruun). Viramo 1992:36.

- fusca (Meig.) Ab: Perniö \& Kiikala. Salmela 2001b:151.

- handlirschi Lacksch. Sb: Savonranta. Oa: Kauhajoki (J. Salmela \& T. Vuorinen). Salmela 2001a:21. Starì \& Salmela 2004:47.

Libnotes ladogensis Lack. $K b$ : Ilomantsi. Polevoi 2001b:191.

Limonia badia (Walk.) Sb: Savonranta, Ob: Rovaniemi. Starì \& Salmela 2004:44.

Ptychoptera hugoi Tjed. Lk: Kolari (K. Mattila). Salmela 2001a:21.

Sycorax silacea Halid. Ab: Kiikala (J. Ilmonen). Salmela 2003b Stud. Dipterol. 10:148.

Ulomyia cognata (Eat.) Ta: Somero (L. Paasivirta) \& Ruovesi (J. Salmela). ibid.:148.

Pneumia [Satchelliella] bucegiana (Vaill.) N: Espoo, $T a$ : Längelmäki, Tampere, Oa: Kauhajoki. Salmela 2002 Diamina 11:31. Salmela 2003b:147.

- stammeri (Jung) Ks: Kuusamo. Salmela 2003b:147.

- trivialis (Eat.) Ab, N, Ta, Sa, Oa, Ks. Salmela 2002:32. Salmela 2003b:147.

- ussurica (Wagn.) Tb: Toivakka. Salmela 2004 Memoranda Soc. Fauna Flora Fenn. 80:6,10.

Pericoma blandula Eat. $L k$ : Kittilä (K. Mattila), $L i$ : Inari (K. Mattila). Salmela 2003b:146.

- formosa Niels. Oa: Kauhajoki (T. Tuovinen \& V. Yli-Teevahainen). ibid.:146.

Tonnoirella nigricauda (Tonn.) Ta: Tampere. Salmela 2002:32. Salmela 2003b:148.

Boreoclytocerus [Clytocerus] rivosus (Tonn.) Ta: Tampere. Salmela 2002:32. Salmela 2003b:145.

- tetracorniculatus Wagn. Ta: Lahti (L. Paasivirta). $T b$ : Toivakka. Salmela 2003b:145. Salmela 2004:10.

Bazarella subneglecta (Tonn.) Oa: Kauhajoki, Ks: Kuusamo, Li: Inari. Salmela 2003b:144.

Psychoda albipennis Zett. Ta, $\mathrm{Sa}$, Oa. Salmela 2002:32. Salmela 2003b:145.

- cinerea Banks Ta: Tampere. Salmela 2003b:147.

- gemina Eat. Ab: Karjaa, Oa: Kauhajoki. ibid:146.

- minuta Banks Ks: Kuusamo. ibid.:147.

Logima satchelli (Quate) $N$ : Esbo, Tb: Toivakka. Salmela 2004:6,10. Salmela 2005 Sahlbergia 10:2.

- erminea Eat. N: Espoo. Salmela 2005:1.

Paramormia polyascoidea (Krek) $\mathrm{Ta}, \mathrm{Oa}, \mathrm{Tb}, \mathrm{Sb}$. Salmela 2003b:146. Salmela 2004:10.

Katamormia niesiolowskii Wagn. Sb: Joroinen. Salmela 2003b:145.

Psycmera integella (Jung) St: Pori, Ta: Tampere, Lahti. Salmela 2002:32. Salmela 2003b:146.

Jungiella consors (Eat.) Ta: Tampere, Luopioinen, $S b$ : Joroinen. Salmela 2002:32. Salmela 2003b:145.

- ellisi With. Ta: Tampere. Salmela 2002:32. Salmela 2003b:145.
- pseudolongicornis Wagn. St, Ta, Sa, Tb. Salmela 2002:32. Salmela 2003b:145. Salmela 2004:10.

Sciria advena (Eat.) Sb: Savonranta. Salmela 2005:2.

Philosepedon balkanicus Krek. Ta: Ruovesi. Salmela 2003b:146.

Dixa dilatata Strobl Ab, N, St, Ta. ibid.:148.

- submaculata Edw. Ab, N, Ta, Oa, Sb, Kb. ibid.: 148 .

Dixella filicornis (Edw.) Sb: Joroinen. ibid.:149.

Bryophaenocladius illimbatus Edw. Ta: Tampere. Salmela 2002:25.

Cricotopus maurii Spies \& Saeth. (polychaetus Hirv. nec Kieff.) Ta: Tampere. ibid.:25.

Georthocladius luteicornis (Goetgh.) Ka: Kymijoki. Raunio \& Langton 2005 Sahlbergia 10:8.

Gymnometriocnemus subnudus (Edw.) Ta: Tampere. Salmela 2002:25.

Limnophyes natalensis (Kieff.) Ta: Tampere. ibid.:25.

Metriocnemus atriclava Kieff. Ta: Tampere. ibid.:25.

- picipes (Meig.) Ta: Tampere. ibid.:25.

Smittia paranudipennis Brund. Ta: Tampere. ibid.:25.

Thienemanniella clavicornis (Kieff.) Kb: Koitajoki. Liljaniemi \& al. 2002 Hydrobiol. 474:250.

Tvetenia discoloripes (Goetgh.) Kb: Koitajoki. ibid.:250.

- paucunca (Saeth.) Kb: Koitajoki. ibid.:250.

Chironomus luridus Strenzke Ta: Riihimäki. Hirvenoja 2002 Sahlbergia 7:7 (only mentioned in the abstract).

- (Lobochironomus) pectoralis (Kieff.) Ta: Tampere. Salmela 2002:25.

Parachironomus tenuicaudatus (Malloch) Ta: Tampere. ibid.:26.

Robackia demeijerei (Krusem.) Kb: Koitajoki. Liljaniemi \& al. 2002:251.

Zavrelliella marmorata (v.d.Wulp) Ta: Tampere. $K b$ : Liperi. Leppä \& Hämäläinen 2001 EF 12:44. Salokannel 2002 Diamina 11:25.

*Stempellina tervolae Gilka Ob: Tervola. Gilka 2005 Ann. Zool. (Warsz.) 55:414.

Tanytarsus pallidicornis (Walk.) Ta: Tampere. Salmela 2002:26.

- palettaris Vern. Ta: Riihimäki. Hirvenoja 2002:12.

Metacnephia saileri (Stone) Li: Inari. Ilmonen 2005 Sahlbergia 10:56.

Hellichiella [Simulium] baffinensis (Twinn) Ta, Lk, $L i$. Ilmonen \& Kuusela 2004 Sahlbergia 8:89.

- crassa Rubts. Ta, Ks, Li. ibid.:89.

Cnetha [Simulium] bicornis Dorog., Rubts. \& Vlas. $O b$ : [Rovaniemi] Meltaus, Ks: Kuusamo. Kuusela 1992 Oulanka Rep. 10:42. Ojanen \& al. 2002 EF 13:157. Yankovsky 2002 Opred. Faune Rossii 170:8.

Thaumalea truncata Edw. Ks: Kuusamo. Salmela 2003a Diamina 12:11. Salmela 2003b:150. Hildén \& al. 2003b:77.

Mycomya simulans Väis. Ta: Heinola (Kaila \& Rus- 
kanen). Polevoi 2001a Entomol. Obozr. 80:519.

- indistincta Pol. Ab: Parainen (P. Chandler). Zaitzev \& Polevoi 1995 EF 6:186.

- humida Garr. Kb: Ilomantsi. Polevoi 2001b:192.

Acnemia angusta Zaitz. [Kb: Ilomantsi,] Tapionaho. Polevoi 2001a:520. Polevoi 2001b:193.

- falcata Zaitz. [Kb: Ilomantsi,] Tapionaho. Polevoi 2001a:520. Polevoi 2001b:193.

- longipes Winn. [Kb: Ilomantsi,] Koitajoki (M. Tietäväinen). Polevoi 2001a:520. Polevoi 2001b:193.

*Leptomorphus subforcipatus Zaitz. \& Ševč. Kb: Ilomantsi (A. Jakovlev). Zaitzev \& Ševčik 2002 Acta Zool. Acad. Sci. Hungar. 48:208.

Monoclona braueri (Strobl) [Kb: Ilomantsi,] Tapionaho. Polevoi 2001a:520. Polevoi 2001b:193.

Phthinia congenita Plassm. [Kb: Ilomantsi,] Tapionaho. Polevoi 2001a:521. Polevoi 2001b:193.

- mira Ostrov. $K b$ : Ilomantsi. Polevoi \& Jakovlev 2004:138.

Sciophila antiqua Chandl. (hebes auct.) Ok: Kuhmo. Komonen 2001 Sahlbergia 6:37. Polevoi \& Jakovlev 2004:138.

- jakutica Blagod. [Kb: Ilomantsi,] Tapionaho. Polevoi 2001a:523. Polevoi 2001b:193.

* - krysheni Polev. Kb: [Ilomantsi,] Tapionaho, Syväjärvi. Polevoi 2001a:523.

Acomoptera difficilis (Dziedz.) Kb: Ilomantsi. Polevoi 2001b:194.

Boletina erythropyga Holmgr. Kb: Ilomantsi. ibid.: 194.

- nitiduloides Zaitz. Kb: Ilomantsi. ibid.:194.

- onegensis Polev. Kb: Ilomantsi. ibid.:194.

- populina Polev. Kb: Ilomantsi. ibid.:194.

* - falcata Polev. \& Hedm. Kb: [Ilomantsi,] Tapionaho, Ok: Kuhmo, Sotkamo. Polevoi \& Hedmark 2004 EF 15:24.

* - hedstroemi Polev. \& Hedm. Ok: Kuhmo. ibid.:25.

* - struthioides Polev. \& Hedm. Le: Kilpisjärvi (L. Tiensuu). ibid.:26.

* - kivachiana Polev. \& Hedm. Ok: Kuhmo. ibid.:27.

* - fennoscandica Polev. \& Hedm. Ok: Kuhmo. ibid.:28.

* - subtriangularis Polev. \& Hedm. Li: Utsjoki (R. Frey). ibid.:30.

* - cordata Polev. \& Hedm. Kb: [Ilomantsi,] Tapionaho, Koitajoki, Ks: Kuusamo (R. Frey). ibid.:31.

- lundbecki Lundstr. (sp. dist.) Lk: Pallastunturi. Polevoi \& Jakovlev 2004 Sahlbergia 9:136.

Impleta consorta Plassm. N: Järvenpää. Kurina 2004 Sahlbergia 8:86.

Syntemna elegantia Plassm. Ok: Kuhmo. Polevoi 2003 Stud. Dipterol. 10:135.

- morosa Winn. Ta, Tb, Kb, Ks. ibid.:137.

* - oulankaensis Polev. Ks: Kuusamo. ibid.:138.

- stylata Huts. Ab, Kb, Ok, Ks. Polevoi 2001b:194. Polevoi 2003:141.
- stylatoides Zaitz. Kb, Ok. Polevoi 2001b:194. Polevoi 2003:141.

Greenomyia baikalica Zaitz. Kb: Ilomantsi. Polevoi 2001b:195.

Tetragoneura ambigua Grzeg. $K b$ : Ilomantsi. ibid.: 195 .

- obirata Plassm. Kb: Ilomantsi. ibid.:195.

Dynatosoma dihaeta Polev. Kb: Ilomantsi. ibid.:195.

- norwegiense Zaitz. \& Økl. Kb: Ilomantsi. ibid.: 195 .

Mycetophila abiecta (Last.) Kb: Ilomantsi. ibid.:195.

- curviseta Lundstr. Kb: Ilomantsi. ibid.:195.

- evanida Last. Kb: Ilomantsi. ibid.:195.

- pseudoforcipata Zaitz. N: Järvenpää. Kurina 2004:86.

- sublunata Zaitz. N: Järvenpää. ibid.:86.

- deflexa Chandl. $K b$ : Ilomantsi. Polevoi \& Jakovlev 2004:137.

* Phronia carli Chandl. (longelamellata auct.) Finland. Chandler 2001 Brit. Journ. Entomol. Nat. Hist. 13:239.

- coritanica Chandl. N: Järvenpää. Kurina 2004:87.

- dubioides Mat. $K b$ : Ilomantsi. Polevoi 2001b:196.

Sceptonia costata (v.d.Wulp) Kb: Ilomantsi. ibid.:196.

- fumipes Edw. Kb: Ilomantsi. ibid.:196.

- fuscipalpis Edw. Kb: Ilomantsi. ibid.:196.

- membranacea Edw. Kb: Ilomantsi. ibid.:196.

Zygomyia kiddi Chandl. Kb: Ilomantsi. ibid.:196.

Allodia embla Hackm. Kb: Ilomantsi. ibid.:197.

- confusa Zaitz. (simplex Zaitz. nec (Buk.)) Kb: Ilomantsi. Polevoi 2001b:197; Zaitzev 2003 Dipterol. Res. 14:88.

- zaitzevi Kur. Kb: Ilomantsi. Polevoi \& Jakovlev 2004:137.

Anatella bremia Chandl. $\mathrm{Kb}$ : Ilomantsi. Polevoi 2001b:197.

- crispa Zaitz. Kb: Ilomantsi. ibid.:197.

- gibba Winn. N: Järvenpää. Kurina 2004:86.

Brachypeza armata Winn. Kb: Ilomantsi. Polevoi 2001b:197.

Brevicornu beatum (Joh.) Kb: Ilomantsi. ibid.:197.

- bipartitum Last.\& Mat. Kb: Ilomantsi. ibid.:197.

- improvisum Zaitz. Kb: Ilomantsi. ibid.:197.

Cordyla nitens Winn. Kb: Ilomantsi. ibid.:197.

- styliforceps (Buk.) Kb: Ilomantsi. ibid.:197.

- murina Winn. Kb: Ilomantsi (previously doubtfully included). ibid.:197.

Exechia pectinivalva Stack. Kb: Ilomantsi. ibid.:198.

Exechiopsis clypeata (Lundstr.) N: Järvenpää. Kurina 2004:86.

*Sciarosoma boreale Chandl. Kb: Lieksa (R. Tuomikoski), Ob: Rovaniemi (R. Tuomikoski). Chandler 2002 Ann. Soc. Entomol. France 38:125.

Sciara nursei Freem. (ulrichi Menz. \& Mohr.) Oa: Ilmajoki. Mukkala \& al. 2005 w-album 2:7; Men- 
zel, Smith \& Chandler 2006 Zool. J. Linn. Soc. 146:134.

Trichosia glabra (Meig.) Tb: Toivakka. Salmela \& Vilkamaa 2005 EF 16:291.

Baeosciara sinuata (Menz. \& Mohr.) N: Helsinki (O. Ranin); Ok: Kuhmo \& Sotkamo (A. Komonen). Vilkamaa \& Komonen 2001:47. Vilkamaa 2003 EF 14:225.

Leptosciarella helvetica (Rudz.) Tb: Konnevesi. Salmela \& Vilkamaa 2005:291.

Cratyna spiculosa (Rudz.) Tb: Konnevesi. ibid.:290.

*Peyerimhoffia infera Vilk. \& Hippa Ta: Lammi (P. Vilkamaa), Om: Nykarleby (W. Hackman). Vilkamaa \& Hippa 2005 Ins. Syst. Evol. 35:473.

* - thula Vilk. \& Hippa Li: Utsjoki (J. Tuiskunen). ibid.:473.

* - menzeli Vilk. \& Hippa Le: Kilpisjärvi (A. Suormala). ibid.:474.

* - sepei Hippa \& Vilk. Lk, Le, Li. Hippa \& Vilkamaa 2005 Zootaxa 1044:51.

Claustropyga corticis (Mohr. \& Ant.) Ks: Salla (E. Pulliainen). Hippa \& al. 2003 Stud. Dipterol. 9:492.

- heteroclausa (Rudz.) Ab, N, Ka, Ok, Ks. ibid.:499.

- subcorticis (Mohr. \& Kriv.) Ks: Iivaara (R. Tuomikoski). ibid.:507.

Corynoptera fera Mohr. \& Hell. Tb: Toivakka. Salmela \& Vilkamaa 2005:290.

- furcifera Mohr. \& Mam. Tb: Toivakka. ibid.:290.

- saetistyla Mohr. \& Kriv. Tb: Toivakka \& Konnevesi. ibid.:290.

- subsedula Mohr. \& Mam. Tb: Rautalampi. ibid.:290.

* Dichopygina aculeata Vilk. \& al. Ab, N, Ta, Kb, Ok, $K s, L e, L i$. Vilkamaa, Hippa \& Komarova 2004 Ins. Syst. Evol. 35:110.

- intermedia (Mohr. \& Kriv.) Ka: Vehkalahti (J. Tuiskunen). ibid.:115.

*-ramosa Vilk. \& al. Ab, N, Ka, Ta, Sa, Om, Ok, Ks. ibid.:119.

Pseudolycoriella brunnea (Buk. \& Leng.) Tb: Toivakka. Salmela \& Vilkamaa 2005:291.

- nodulosa (Mohr. \& Kriv.) Tb: Toivakka. ibid.:291.

Lycoriella micria Mohr. \& Menz. Tb: Rautalampi. ibid.:291.

- autostylia Mohr. \& Menz. Oa: Ilmajoki. Mukkala \& al. 2005:7.

- ingenua (Duf.) (solani (Winn.), brevipila Tuomik.) Finland. Menzel \& Mohrig 1999 Stud. Dipterol., Suppl. 6:393.

*Ctenosciara exigua Salm. \& Vilk. Oa: Kauhajoki, Tb: Toivakka. Salmela \& Vilkamaa 2005:297.

Bradysia subbetuleti Mohr. \& Kriv. Tb: Konnevesi, Rautalampi. ibid.:290.

- submoesta Mohr. \& Kriv. Tb: Toivakka. ibid.:290.
- subscabricornis Mohr. \& Menz. Tb: Toivakka. ibid.:290.

Scaptosciara neglecta Menz. \& Mohr. Tb: Toivakka, Konnevesi. ibid.:291.

Epidosis modesta (Spung.) Ta: Lammi (J. Jakovlev). Penttinen \& Siitonen 2005 Sahlbergia 10:52.

Monepidosis pectinata Mam. Tb: Keuruu. ibid.:52.

Pseudepidosis trifida Mam. Tb: Toivakka. ibid.:53

Paratetraneuromyia vernalis Spung. Tb: Toivakka \& Laukaa. ibid.:52

Porricondyla lutescens Spung. $T b$ : Rautalampi. ibid.:52.

Contarinia vincetoxici Kieff. $A l, A b$. Widenfalk \& al. 2002 Syst. Entomol. 27:520.

Dasineura pustulans (Rübs.) Finland. Huldén 2004 Sahlbergia 8:100.

Ectaetia clavipes (Loew) Ab: Vichtis (R. Frey). Krivosheina 2002 Entomol. Obozr. 81:735.

Thripomorpha paludicola End. $N$ : Hangö, Helsinge. Krivosheina 2001 Zool. Zhurn. 80:549.

Swammerdamella adercotris Cook Al: Saltvik (R. Frey), $A b$ : Nystad (W. Hellén). ibid.:552.

Sylvicola stackelbergi Kriv. \& Menz. N: Grankulla (P. Grotenfelt) \& Helsingfors (T. Clayhills \& L. Tiensuu), St: Tyrvää (P. Savolainen). Krivosheina \& Menzel 2002 Entomol. Obozr. 81:426.

Xylophagus matsumurae Miyat. Tb: Saarijärvi, Konginkangas. Kahanpää \& Winqvist 2005:13.

Rhagio notatus (Meig.) N, Ta. ibid.:13.

Sargus flavipes Meig. (splendens auct.). Finland. ibid.: 20.

Tolmerus pyragra Zell. Sa: Luumäki. ibid.:13.

Villa modesta (Meig.) Al, Ab, N, Ta, Sa, Kl. Kahanpää \& Winqvist 2005:14.

Microphor anomalus (Meig.) Al: Mariehamn (R. Frey). ibid.:17.

Platypalpus annulipes (Meig.) Ab: Turku (K. Winqvist). Hildén \& al. 2003b:77. Kahanpää \& Winqvist 2004 Sahlbergia 8:92.

- scandinavicus Chvála Oa: Ilmajoki. Mukkala \& al. 2005:7. Kahanpää \& Winqvist 2005:17.

- fuscicornis (Zett.) N: Espoo. Kahanpää \& Winqvist 2005:17.

- albicornis (Zett.) Al, Ab. Chvála 1975 Fauna Ent. Scand. 3:195. Accidentally omitted from check list in 1980 (Hackman 1980 NE 60).

Hilara sturmii Wied. Ab, N, St. Chvála 2005 Fauna Ent. Scand. 40:51.

- eviana Straka Sb. ibid.:73.

- hyposeta Straka Ta, Sa, Kb. Kahanpää \& Winqwist 2005:15. Chvála 2005:75

- hirta Strobl Ab, N, Sa, Lk. Chvála 2002b Acta Univ. Carol. Biol. 46:244. Chvála 2005:113.

- coracina Old. Al, Ab, N. Chvála 2002a EF 13:75. Chvála 2005:139.

- longifurca Strobl Ab, N, Ka, Sb, Om. Kahanpää \& Winqvist 2005:15 Chvála 2005:143.

- campinosensis Nies. $A l, A b, S t, K b$. Kahanpää \& 
Winqvist 2005:15. Chvála 2005:145.

- implicata Coll. Al, Ab, N, St. Kahanpää \& Winqvist 2005:15. Chvála 2005:147.

* - lapponica Chvála Li: Inari, Utsjoki. Chvála 2002a EF 13:69. Chvála 2005:173.

* - quadrifasciata Chvála (quadrivittata auct.). Al, $\mathrm{Ab}, \mathrm{N}, \mathrm{Ta}, \mathrm{Sa}, \mathrm{Oa}, \mathrm{Sb}, \mathrm{Kb}, \mathrm{Om}, \mathrm{Ks}$. Chvála 2002

Acta Univ. Carol., Biol. 46:257. Kahanpää \& Winqvist 2005:23.

- woodiella Chvála $A b$ : Åbo (R. Frey), N: Esbo (B. Poppius). Kahanpää \& Winqvist 2005:16. Chvála 2005:179.

- biseta Coll. N: Kyrkslätt (R. Frey), Sb: Maaninka (C. Lundström). Kahanpää \& Winqvist 2005:15. Chvála 2005:191.

- albitarsis Ros. Ab, Ta, Sa. Kahanpää \& Winqvist 2005:15. Chvála 2005:218.

Rhamphomyia cinerascens (Meig.) Sb: Kuopio (C. Lundström). Kahanpää \& Winqvist 2005:16.

- laevipes (Fall.) Sa, Sb. ibid.:16.

- aethiops Zett. (caudata auct. nec (Zett.)) Finland. ibid.:16.

- pilifer Meig. (dentipes Zett., intermedia Frey) Finland. ibid.:24.

- helleni Frey Lk: Muonio (R. Frey). ibid.:16.

- trigemina Old. Ab: Pargas (R. Frey). ibid.:16.

- galactoptera Strobl Sa: Joutseno (E. Thuneberg). ibid.:16.

Hemerodromia adulatoria Coll. Ks: Kuusamo (R. Frey). ibid.:16.

Rhaphium zetterstedti Par. Ab: Pertteli (A. Haarto \& V.-M. Mukkala). Haarto 2002b Sahlbergia 7:36.

Syntormon denticulatum (Zett.) Ab: Kiikala. Accidentally omitted from check list in 1980 (Hackman 1980 NE 60), cf. Frey 1915 Acta Soc. Fauna Flora Fenn. 40(5):41. Haarto 2002b:36.

Dolichopus austriacus Par. N: Pyhtää (V.-M. Mukkala). Haarto 2002b:36.

Dolichophorus kerteszi Lichtw. Ta: Hartola, Sysmä. Kahanpää \& Winqvist 2004:92.

Diplonevra concinna (Meig.) Sa: Puumala. ibid.:93.

Megaselia opacicornis Schmitz Li: Utsjoki Kevo. Gross \& al. 2004 Ecol. Entomol. 29:588.

Spiniphora maculata (Meig.) Ta: Hartola. Kahanpää \& Winqvist 2004:93.

Paraplatypeza bicincta (Szil.) N: Helsinki (R. Tuomikoski), St: Yläne (J. Sahlberg). Chandler 2001 Fauna Ent. Scand. 36:234.

Protoclythia modesta (Zett.) Al. ibid.:234.

Dasysyrphus friuliensis v.d.Goot $N, K s, L k, L i$. Haarto \& Kerppola 2004 Sahlbergia 9:150.

Epistrophe annulitarsis (Stack.) Tb: Pihtipudas. Piirainen 2001 Diamina 10:1.

- diaphana (Zett.) N: Helsinki (T. Järveläinen). Haarto \& Kerppola 2004:150.

Leucozona inopinata Docz. Ab, $\mathrm{N}, \mathrm{Ka}, \mathrm{St}, \mathrm{Ta}, \mathrm{Sa}, \mathrm{Kl}$, $\mathrm{Oa}, \mathrm{Sb}, \mathrm{Om}$. ibid.: 150 .
Eupeodes curtus (Hine) Li: Inari (J. Kahanpää). ibid.: 150 .

Parasyrphus groenlandicus (Niels.) Le: Kilpisjärvi. Haarto 2002a Sahlbergia 7:29; Haarto 2002b:36.

- proximus Mut. $N$ : Helsinki (S. Kerppola). Haarto \& Kerppola 2004:150.

- punctulatus (Verr.) Al, Ab, N, Ka, Ta. ibid.:150.

Sphaerophoria abbreviata Zett. $A b, T a, O k, K s, L k$, Li. ibid.:151.

Syrphus rectus O-Sack. N: Helsinki (E. Räsänen \& Hölttä). ibid.:151.

Chrysotoxum octomaculatum Curt. Ab, Kl. ibid.:150.

Platycheirus europaeus Goeld., Maib. \& Sp. Ab, $N$, $\mathrm{Ta}, \mathrm{Sa}, \mathrm{Oa}, \mathrm{Sb}, \mathrm{Kb}, \mathrm{Ok}, \mathrm{Ks}, \mathrm{Lk}, \mathrm{Le}, \mathrm{Li}$. ibid.: 149 .

- laskai Niels. Ab, N, Sa, Oa, Ok. ibid.:149.

- lundbecki (Coll.) Le: Kilpisjärvi (W. Hellén). ibid.:149.

- parmatus Rond. Ks: Oulanka(G. Ståhls), Lk: Savukoski (A. Haarto). ibid.:149.

- perpallidus Verr. N, Ob, Lk, Le, Li. ibid.:149.

- ramsarensis Goeldl., Maib. \& Sp. Le: Enontekiö. Haarto 2002a:30; Haarto 2002b:35.

- splendidus Roth. Al: Finström (R. Frey). Haarto \& Kerppola 2004:149.

- subordinatus Beck. Le: Enontekiö. Haarto 2002a:30; Haarto 2002b:35

- tarsalis (Schumm.) Al: Eckerö (E. \& L. Laasonen), Ks: Oulanka (G. Ståhls). Haarto \& Kerppola 2004:149.

- varipes Curr. $L k$ : Muonio (J.A. Palmén), Li: Utsjoki (E. \& L. Laasonen). ibid.:149.

Pipiza festiva Meig. N: Helsinki (S. Kerppola). ibid.:153.

Pipizella certa Viol. N, Ka, Ta. ibid.:153.

Trichopsomyia joratensis Goeldl. Ta: Längelmäki (K. Mattila). Haarto \& Kerppola 2004:153. Piirainen \& Mattila 2005 Diamina 14:2.

Triglyphus primus Loew $A b$ : Turku (A. Haarto), $N$ : Helsinki (S. Kerppola). Haarto \& Kerppola 2004:153.

Cheilosia alba Vuj. \& Cl. Ab Suomusjärvi (J. Kahanpää), Ta: Somero (A. Haarto), ibid.:151.

- impressa Loew Ab, N, St, Ta, Sa, Tb, Sb. ibid.:151.

* - ingerae Niels. \& Cl. Lk: Kittilä. Nielsen \& Claussen 2001 Dipteron 4:43. Haarto \& Kerppola 2004:151.

- lasiopa Kow. Finland. ibid.:160.

- morio (Zett.) Ab, N, Ta, Ok. ibid.:151.

- psilophthalma Beck. Ab, N, Ta. ibid.:151.

- urbana Meig. Ab, N, St, Ta, Sa, Om, Ob. ibid.:151.

Brachyopa obscura Thomp. \& Torp N: Helsinki, Vantaa. ibid.:151.

Chrysogaster virescens Loew N: Helsinki (S. Kerppola). ibid.:152.

Chrysosyrphus nasutus (Zett.) Ks, Lk, Le, Li. ibid.: 152 . 
Orthonevra plumbago (Loew) $N$ : Helsingfors (W. Nylander), $S b$ : Kuopio (C. Lundström). ibid: 152.

- stackelbergi Thomp. \& Torp Ab, N, St, Ta, Ob, Ks. ibid.: 152 .

Neoascia obliqua Coe $A b$ : Kaarina, $N$ : Inkoo. ibid.: 152.

Sericomyia jakutica (Stack.) Li: Utsjoki (R. Frey \& W. Hellén). ibid.153.

Eristalis alpina (Panz.) Finland. Hippa, Nielsen \& Steenis 2001 Norw. J. Entomol. 48:294.

- pseudorupium Kan. (sp. dist.) Ta, Li. ibid.:308.

Helophilus bottnicus Wahlb. Ok: Ruhtinansalmi (O. Sorsakoski). Haarto \& Kerppola 2004:152.

Temnostoma angustistriatum Kriv. (bombylans auct.). $N, K a, S t, T a, T b, S b$. Krivosheina \& Ståhls 2004 Sahlbergia 8:97.

- carens Gaun. (sp. dist.) Ok: Ruhtinansalmi (O. Sorsakoski). Krivosheina 2003 Zool. Zhurn. 82:1480. Haarto \& Kerppola 2004:153.

- sericomyiaeforme (Port.) (sp. dist.) N: Esbo (Pipping), $S a$ : [Joutseno] Rajala (Redikotzev). Krivosheina \& Bagachanova 2004 Zool. Zhurn. 83:75.

Xylota jakutorum Bagatsch. Finland. Haarto 2002b:35. Bartsch, Nielsen \& Speight 2002 Volucella 6:77.

Eudorylas coloratus (Beck.) (fascipes auct. nec (Zett.)). Finland. Kehlmaier 2005 Verh. Naturwiss. Ver. Hamburg 41:221.

- goennersdorfensis Dempew. \& Dunk Ta: Lammi (F. Mihályi). ibid.:246.

Pipunculus zugmayeriae Kow. Ab: Turku. Kahanpää \& Winqvist 2004:93.

Thecophora pusilla (Meig.) N: Helsinki. ibid.:93.

Sepsis luteipes Mel. \& Spul. (sp. dist.) Ab-Lk. Pont \& Meier 2002 Fauna Ent. Scand. 37:169.

Palloptera venusta Loew $K b$ : Ilomantsi. Polevoi 2001b:202.

- marginata (Meig.) Ta: Somero (T. Mutanen). Haarto 2002b:36.

* Lonchaea fraxina McGow. \& Roth. Al: Saltvik (R. Frey). MacGowan \& Rotheray 2000 Dipter. Dig. $7: 38$.

* - caledonica McGow. \& Roth. (laticornis auct. nec Meig.) Finland. ibid.:40.

*Agromyza quadriseta Zlob. N: Helsinge (R. Frey). Zlobin 2001 Dipterol. Res. 13:92.

Phytomyza pubicornis Hend. Ab: Turku. Kahanpää \& Winqvist 2004:93.

Amiota rufescens (Oldenb.) Ob: Oulu. Baechli \& al. 2004 Fauna Ent. Scand. 39:50.

* Drosophila vireni Bch., Vil. \& Har. Ks: Kuusamo Oulanka (S. Lakovaara). Bächli \& al. 2002 Mitt. Schweiz. Ent. Ges. 75:312.

Hirtodrosophila confusa (Staeg.) N: Helsinki. Baechli \& al. 2004:248.

- oldenbergi Duda Sa: Puumala. Kahanpää \& Winqvist 2004:94.
Microdrosophila congesta (Zett.) Al, N. Baechli \& al. 2004:270, 347.

Scaptomyza montana Wheel. $A l, A b, N, K a, T a, O b$, $K s, L i$. ibid.:309, 347.

Elachiptera diastema Coll. $A b, N, K a$. ibid.:94.

Dicraeus vagans (Meig.) Ab: Turku. ibid.:93.

* Pullimosina vulgesta Roh. (moesta auct. nec (Villen.)). Rohaček 2000 Bull. Soc. Entomol. France 105:474.

Clusiodes microcerca Stack. Kb: Ilomantsi. Polevoi 2001b:202.

*Trimerina indistincta Kriv. Ab: Lojo (W. Hellén), $N$ : Grankulla (R. Frey), Ta: Orivesi (L. Tiensuu). Krivosheina 2004 Zool. Zhurn. 83:631.

Notiphila major Stenh. Lk: Sodankylä. Kahanpää \& Winqvist 2004:94.

Ditrichophora pulchella (Meig.) Ab: Turku. Winqvist 2002 Sahlbergia 7:37.

Parydra arctica Claus. Oa: Solf (Håk. Lindberg), Li: Utsjoki (R. Frey). Krivosheina 2000 Dipterol. Res. 11::173.

Coenia curvicauda (Meig.) Ab: Turku. Winqvist 2002:36.

Calocoenia paurosoma (Sturt. \& Wh.) Le: Kilpisjärvi (R. Storå). Krivosheina 2000:172.

Chlorops kirigaminensis Kanm. (zonulatus auct.) Al, $A b, N, T a, S a, K 1, S b, K s$. Nartshuk 2002 Diptero1. Res. 13:17.

Cosmetopus longus (Walk.) (bergrothi Beck.) (sp. dist.) Finland. Gorodkov 1986 Catal. Pal. Dipt. 11:23.

Coniosternum lapponicum Ringd. Finland. Šifner 2003 Acta Mus. Natn. Prag., Ser. B 59:78.

Fannia lucidula (Zett.) (= F. glaucescens auct.) (sp.dist.). Pont 2002 Ins. Syst. Evol. 33:107.

Coenosia means Meig. Ob, Lk, Li. Kahanpää \& Winqvist 2004:94.

- lineatipes (Zett.) Oa: Ilmajoki. Mukkala \& al. 2005:7.

Alliopsis [Paraprosalpia] denticauda Zett. Finland. Tiensuu 1933 Notulae Entomol. 13:79 (accidentally omitted from check list in 1980).

Alliopsis conifrons (Zett.) $\mathrm{Ob}, \mathrm{Ks}, \mathrm{Lk}, \mathrm{Le}, \mathrm{Li}$. Kahanpää \& Winqvist 2004:94.

Nemorilla maculosa (Meig.) Al: Kökar (J. Itämies). Haarto 2002b:35.

Ramonda prunaria (Rond.) Ta: Somero (A. Haarto \& V.-M. Mukkala). ibid.:35.

Present number: 5,349 $-103+305=5,551$ spp.

\section{Hymenoptera}

Koponen, M., Jussila, R. \& Vikberg, V. 2003. Suomen loispistiäisluettelo (Hymenoptera, Parasitica). Osa 5, heimo Ichneumonidae, alaheimot Tersilonchinae, Ophioninae, Paxylommatinae, Cremastinae ja Campopleginae. - Sahlbergia 8:2748. 
Söderman, G. \& Leinonen, R. 2003. Suomen mesipistiäiset ja niiden uhanalaisuus. - Tremex Press, Helsinki, 420 pp.

Söderman, G. \& Vikberg, V. 2003. Suomen myrkkypistiäisten luettelo ja levinneisyys (Hymenoptera, Apocrita, Aculeata). - Sahlbergia 7:41-66.

Yu, D.S., van Achterberg, K. \& Horstmann, K. 2005. World Ichneumonoidea 2004. Taxonomy, Biology, Morphology and Distribution. CD/DVD. Taxapad, Vancouver, Canada. [with species numbers]

Deletions

Aprosthema bifidum (Klug) (=A.melanurum (Klug)) Zhelochovtsev \& Zinovjev 1995 Entomol. Obozr. 74:401.

Eupontania lapponica (Mal.) $(=$ E. crassipes (Thoms.)). Vikberg 2003 EF 14:135.

Phytodietus crassitarsis Thoms. $(=$ P. arcuatorius (Thunb.)). Yu \& al. 2005:63845.

- segmentator Grav. (= P. polyzonias (Forst.)). ibid.:63903.

Polyblastus affinis Woldst. (= P. varitarsus (Grav.)). ibid.:64522.

Exenterus claripennis Thoms. $(=$ E. amictorius (Panz.)). ibid.:63013.

Charitopes brunneus (Morl.) $(=C$. areolaris (Thoms.)). ibid.:39383.

Sulcarius hellbachi (Schmied.) (= S. biannulatus (Grav.)). ibid.:41999.

Phygadeuon oppositus Thoms. (= P. subtilis Grav.). ibid.:41690.

Cubocephalus erythrinus (Grav.) (=C. sperator (Müll.)). Sawoniewicz 2003 Entomofauna 24:214.

Baeosemus vulpecula Holmgr. (= B. mitigiosus (Grav.)). Yu \& al. 2005:45405.

Trachyarus specularis Rom. (= T. fuscipes Thoms.). ibid.:46029.

Cratichneumon fabricator (F.) (= C. culex (Müll.)). ibid.:48386.

Ichneumon latrator F. (f.d., = I. oblongus Schrank). Horstmann 2001 Beitr. Entomol. 51:40.

- versutus Holmgr. (= I. vulneratorius Zett.). Yu \& al. 2005:51100.

Plectiscus flavicornis (Thoms.) (= P. agilis (Holmgr.)). ibid.:58286.

Plectiscidea humilis (Först.) (= Proclitus zonatus (Grav.). ibid.:58376.

Proclitus absconditus Först. (= P. zonatus (Grav.). ibid.:58372.

Helictes pumilio (Först.) (= Megastylus orbitator Schte) ibid.:57972.

- rufipleuris (Först.) (= Megastylus orbitator Schte) ibid.:57974

Tromatobia oculatoria (F.) (f.d., = T. lineatoria (Vill.)). Horstmann 2001:42.
Schizopyga varipes Holmgr. (= S. podagrica Grav.). Yu \& al. 2005:59996.

Pimpla padellae Torka (= P. turionellae (L.)). ibid.:61171.

Ctenopelma luteum Holmgr. (= C. tomentosum (Desv.)), ibid.:42383.

- xanthostigma Holmgr. $(=C$. tomentosum (Desv.)). ibid. :48384.

Himerta varicornis (Grav.) (=H. defectiva (Grav.)). ibid.:43280.

Campodorus humerellus (Thoms.) (= C. scapularis (Steph.). ibid.:43227.

Mesoleius stenocerus (Thoms.) (= M. hyperboreus Holmgr.). Kasparyan 2001 Ent. Obozr. 80:713.

- furax Holmgr. (= M. opticus (Grav.)). ibid.:707,710.

- sinuatus Thoms. (= M. intermedius (Grav.)). Yu \& al. 2005:43504.

Mesoleptidea amoena (Holmgr.) (= M. cingulata (Grav.)). ibid.:42723.

Dimophora nitens (Grav.) (robusta Brischke) Recorded outside Finland. Koponen \& al. 2003:31.

Cremastus geminus Grav. Recorded outside Finland. ibid:31.

Aneuclis affinis Hellén (= Tersilochus filicornis (Thoms.)). ibid.:33.

- incidens (Thoms.) Recorded outside Finland. ibid:31.

Campoletis vexans (Holmgr.) $(=$ C. viennensis (Grav.)). ibid.:40.

Dusona annexa (Först.) (f.d., = D. leptogaster (Holmgr.). ibid.:38.

- oxyacanthae (Boie) Recorded outside Finland. ibid:31.

Astiphromma plagiatum (Thoms.) (=A. splenium (Curt.)). Yu \& al. 2005:53974.

- strenuum (Holmgr.) (= A. splenium (Curt.)). ibid.:53972.

Agrypon brachycerum Hellén ( $=$ A. anxium (Wesm.)). Koponen \& al. 2003:35.

- delarvatum (Grav.) Recorded outside Finland. ibid:31.

Dendrosoter flaviventris Först. (= D. hartigii (Ratz.)). Belokobylskij \& al. 2003 Beitr. Entomol. 53:376.

Oncophanes caudalis Hellén (= Ontsira antica (Woll.)). Yu \& al. 2005:12797.

Rhysipolis varicoxa (Thoms.) (= R. meditator (Hal.)). ibid.:23173.

Opius hilaris Fisch. (= O. posticatae Fisch.). Belokobylskij \& al. 2003:396.

- seebensteinensis Fisch. (= O. posticatae Fisch.). ibid.:396.

- minor Fisch. (=O. gracilis Fisch.). ibid.:394.

- basirufus Fisch. (=O similis Szépl.). ibid.:396.

- altimontanus Fisch. (=O. similis Szépl.). ibid.:396.

- piceus Thoms. (= O. pallipes Wesm.). ibid.:395. 
- meracus Fisch. (=O. pygmaeator (Nees)). ibid.:395.

- funebris Wesm. (= O. pygmaeator (Nees)). ibid.:395.

- latipes Fisch. (= O. pendulus Hal.). ibid.:395.

- reconditor Wesm. (f.d., $=O$. recondens (Acht.). van Achterberg 2004 Zool. Meded. Leiden 78:334.

Alysia curtungula Thoms. (= A. manducator (Panz.)). Yu \& al. 2005:1691.

Coelinius anceps (Curt.) $(=$ C. parvulus (Nees)). Belokobylskij \& al. 2003:360.

- $\operatorname{circulator}(\mathrm{Grav}).(=$ C. parvulus (Nees)). ibid.:360.

Chelonus catulus Marsh. (= Ascogaster varipes Wesm.). Yu \& al. 2005:10902.

- rimulosus Thoms. (=C. sulcatus Jur.). ibid.:11806.

Microgaster acilius Nixon (= M. meridianus Hal.). ibid.:19791.

Trioxys hincksi Mack. (= T. betulae Marsh.). ibid.:4880.

Adialytus arvicola Stary (= A. ambiguus (Hal.)). Belokobylskij \& al. 2003:364.

Taphaeus filator (Nees) (=Diospilus capito (Nees)). Yu \& al. 2005:16297.

Perilitus secalis (Hal.) (= P. cerealium (Hal.)). ibid.:15629.

Eubazus opacus Reinh. (=Foersteria puber (Hal.)). Belokobylskij \& al. 2003:368.

Bassus cingulator (Ratz.) $(=B$. dimidiator Nees). ibid.:355.

Copidosoma auricollis (Thoms.) (= C. agrotis (Fonsc.)). Guerreri \& Noyes 2005 Syst. Ent. 30:111.

Laelius femoralis (Först.) (f.d., = L. borealis Vikb.). Vikberg \& Koponen 2005 EF 16:32.

Myrmica microrubra Seif. (= M. rubra (L.)). Savolainen \& Vepsäläinen 2003 Proc.Nat.Acad.Sci. 100:7172.

Lasius alienus (Först.) (f.d., = L. psammophilus Seif.). Söderman \& Vikberg 2003:60.

Arachnospila nivalabnormis (Wolf) (f.d., = A. spissa (Schte.)). ibid.:61.

Osmia ventralis (Panz.) $(=$ O. leaiana (Kby.). ibid.:62. Söderman \& Leinonen 2003:212.

\section{Additions}

*Pamphilius kontuniemii Shinoh. Ab, N, Ka, Ta, Sa, $\mathrm{Kl}, \mathrm{Tb}, \mathrm{Sb}, \mathrm{Kb}, \mathrm{Om}, \mathrm{Ok}, \mathrm{Ob}, \mathrm{Ks}, \mathrm{Lk}, \mathrm{Li}$. Shinohara 2003 Ins. Syst. Evol. 34:463.

Dolerus brevicornis Zadd. (sp. dist.). Finland (north to $\mathrm{Li}$ ). Heidemaa, Nuorteva, Hantula \& Saarma 2004 Eur. Journ. Ent. 101:647.

Sterictiphora sorbi Kontun. (sp. dist.). N, Tb, Sb, Ks. Nuorteva \& Nuorteva 2003 EF 14:173.
*Euura boreoalpina Kop. Le: Kilpisjärvi. Kopelke 2001 Senckenb. Biol. 81:197.

- hastatae Mal. (sp. dist.) Le: Kilpisjärvi. ibid. 213.

- lappo Mal. (sp. dist.) Le: Kilpisjärvi. ibid. 216.

* - phylicifoliae Kop. Le: Kilpisjärvi. ibid. 220.

Eupontania crassipes (Thoms.) (sp. dist.). Lk, Le. Vikberg 2003 EF 14:135.

- myrsiniticola (Kop.) Le: Kilpisjärvi. ibid.:157.

Pontania triandrae Bens. (sp. dist.). Kopelke 2005 Senckenberg. Biol. 85:92.

Thymaris collaris Thoms. (sp. dist.). Yu \& al. 2005:63352.

Ctenochira breviseta (Ratz.) " Finnland (Coll. Jussila)”. Horstmann 2002 Mitt. Münch. Ent. Ges. 92:83.

Xorides praecatorius (F.) Ab: Nauvo (I. Sääksjärvi). Jussila \& al. 2003 Sahlbergia 8:22.

Helcostizus maculatus (Woldst.) (sp.dist.) Ob: Kolari (J. Siitonen). Sawoniewicz 2003 Entomofauna 24:219.

Atractodes alpestris Rom. Sa: Mikkeli (M. Koponen). Jussila 2001 EF 12:193.

- tenuipes Thoms. Ab, N, Ka, St, Kb, Om, Ks. ibid.:198.

- remotus Juss. (sp. dist.) $O b$ : Ii, $L k$ : Kolari. ibid.:200.

Pleolophus cursitans Kriechb. Kb: Ilomantsi. Humala 2001 Metsähall. luonnonsuoj.julk. A 131:206.

Aoplus biannulatorius (Thunb.) (pulchricornis (Grav.)) $K b$ : Ilomantsi. ibid.:210.

Ichneumon erythromerus Wesm. (corfitzi Thoms.) (sp. dist.). Horstmann 2003 Mitt. Münch. Ent. Ges. 93:26.

- oblongus Schrank (latrator auct. nec F.). Horstmann 2001 Beitr. Entomol. 51:40.

*Cylloceria tenuicornis Hum. Ab: Karislojo (W. Hellén), Ta: Tavastehus (J. Sahlberg), Li: Ivalo. Humala 2002 Entomol. Obozr. 81:380.

Aniseres caudatus Hum. $\mathrm{Kb}$ : Ilomantsi. Humala 2001:209.

*Allomacrus subtilis Hum. Kb: [Ilomantsi], Pieni Kotavaara (M. Tietäväinen). Humala 2002:381.

Orthocentrus radialis Thoms. Kb: Ilomantsi. Humala 2001:209.

Gnathochorisis crassulus (Thoms.) (sp. dist.). Yu \& al. 2005:57870.

- xanthocephala (Strobl) Finland. Humala 2003 Finn. Environm. 485:196.

Eusterinx inaequalis Ross. $K b$ : Ilomantsi. Humala 2001:208; Humala 2004 Trudy Russ. Ent. Obshch. 75:67.

- circaea Ross. Finland. Humala 2004:67.

- trifasciata (Ashm.) Finland. ibid.:68.

- aquilonigena Ross. Finland. ibid.:68.

- minima (Strobl) Finland. ibid.:69.

- refrectaria Ross. Finland. ibid.:70.

Plectiscidea fuscifemur Hum. Finland. Humala 2003:196. 
- koponeni Hum. Finland. ibid.:196.

- helleni Hum. Finland. ibid.:196.

Hemiphanes performidatum Ross. Finland. ibid.:196.

Helictes carinatus Hum. Finland. ibid.:196.

Megastylus orbitator Schte. (pumilio (Först.), rufipleuris (Först.)). Yu \& al. 2005:57948.

Proeliator proprius Ross. Finland. Humala 2003:196.

Delomerista strandi Ulbr. $K b$ : Ilomantsi. Humala 2001:205.

Scambus signatus (Pfeff.) Al, Ab, St, Oa, Om. Jussila \& al. 2003 Sahlbergia 8:21.

- tenthredinum (Gze.) Ab: Pyhäjärvi (V. Vikberg). Horstmann 2005 Entomofauna 26:108.

Polysphincta nielseni Rom. Oa: Kristinestad. Fritzén 2005 Memoranda Soc. Fauna Flora Fenn. 81:49.

Tromatobia lineatoria (Vill.) (oculatoria auct. nec (F.). Horstmann 2001:42.

Ctenopelma tomentosum (Desv.) (luteum Holmgr., xanthostigma Holmgr.). Yu \& al. 2005:12382.

Lethades cingulator Hinz Ab: Sauvo. Jussila 2001 EF 12:119.

- curvispina (Thoms.) Ab, Ta, Oa, Ks, Li. ibid.:119.

- laricis Hinz Li: Utsjoki (S. Koponen \& E. Linnaluoto). Jussila \& al. 2003:22.

Trematopygus helleni Hinz Lk: Kolari, Li: Utsjoki. Jussila 2001:119.

- rufator Hinz Li: Utsjoki. ibid.:120.

*Campodorus nigriventris Kasp. Le: Kilpisjärvi (R. Hinz). Kasparyan 2005 Ent. Obozr. 84:189.

Rhorus chrysopygus (Rom.) Li: Utsjoki. Jussila 2001:120.

Cremastus pungens Grav. Ab, $N, \mathrm{Ka}$, St, Tb. Koponen \& al. 2003:28.

Diaparsis frontella (Holmgr.) Finland. ibid.:32.

- rarus (Horstm.) Finland. ibid.:32.

Aneuclis maritima (Thoms.) Ab: Vihti (V. Karvonen). ibid.:27.

Epistathmus crassicornis Horstm. Finland. ibid.:32.

Barycnemis angustipennis (Holmgr.) (sp. dist.) Finland.ibid.:32.

- filicornis (Thoms.) Ab, N, Ka, Ta, Sa, Kb, Tb, Om, Ok, Ob, Ks. ibid.:27.

- punctifrons Horstm. Ab: Turku (R. Jussila). ibid.:28.

Probles longicaudator Aub. Finland. ibid.:33.

Tersilochus spiracularis Horstm. Finland. ibid.:33.

Sinophorus juniperinus (Holmgr.) (sp. dist.). Finland. ibid.:36.

Campoplex scaposus (Thoms.) Ab, N, Oa. ibid.:28.

Nemeritis aequalis Horstm. Ab: Houtskär (H. Bruun). ibid.:29.

- breviventris Horstm. Ab: Tenhola (R. Jussila). ibid.:29.

Bathyplectes balteatus (Thoms.) Ab, N, Oa. ibid.:29.

- immolator (Grav.) Ab: Turku, Ks: Oulanka. ibid.:29.

Campoletis cognata (Tschek) Finland. ibid.:39.
Dusona contumax (Först.) Ab, N, Ka, Tb, Sb, Om, $\mathrm{Li}$. ibid.:28.

- incompleta (Bridgm.) Al, Ab, N. ibid.:29.

- subimpressa (Först.) N: Helsinki (J. Perkiömäki). ibid.:29.

Phobocampe alticollis (Thoms.) Finland. ibid.:40.

- crassiuscula (Grav.) Ab, N. ibid.:29.

- flavicincta (Thoms.) Ab, Ka, Li. ibid.:29.

- luctuosa Schmied. Ab, N, Ka. ibid.:29.

Tranosema nigridens (Thoms.) Ab, Ta. ibid.:29.

Diadegma aculeatum (Bridgm.) N: Sibbo (A. Albrecht). ibid.:29.

- albotibiale Horstm. Finland. ibid.:40.

- anurum (Thoms.) Ka, Om, Ok. ibid.:29.

- consumptor (Grav.) Ab, N, Ka, St, Ta, Sa, Oa, Om, Le, Li. ibid.:29.

- contractum (Brischke) N: Helsinki (V. Karvonen). ibid.:29.

- crassiseta (Thoms.) Ab: Korppoo (R. Jussila). ibid.:29.

- elishae (Bridgm.) Li: Utsjoki (R. Jussila). ibid.:29.

- elongatum (Thoms.) Ab, N, Le. ibid.:29.

! - exareolator Aub. $K b$ : Ilomantsi. Humala 2001:208.

- gibbulum (Brischke) Ab, N, Ka. Koponen \& al. 2003.:29.

- holopygum (Thoms.) Al, N, St, Sa. ibid.:30.

- maculatum (Grav.) Ab, N, Ka. ibid.:30.

- melanium (Thoms.) Al, Ab, N, Ka. ibid.:30.

- pulicator Aub. Ab: Turku (R. Jussila). ibid.:30.

- rufatum (Bridgm.) Ab, N, Li. ibid.:30.

- speculare (Thoms.) Ab: Mietoinen, Sauvo. ibid.:30.

- stigmatellae Horstm. Ab, Ka, Oa. ibid.:30.

- trochanteratum (Thoms.) (sp. dist.) Finland. ibid.:41.

Hyposoter albonotatus (Bridgm.) Ab, N, St, Oa, Om. ibid.:30.

- coxator (Thoms.) N, Sa. ibid.:30.

- discedens (Schmied.) Ab, Sa, Oa, Ob, Li. ibid.:30.

- fitchii (Bridgm.) Ab, N. ibid.:30.

- longulus (Thoms.) Ab, N, Ka, Ta, Sa. ibid.:30.

- pectinatus (Thoms.) Finland. ibid.:41.

- virginalis (Grav.) N: Tammisaari (V. Karvonen). ibid.:30.

- vividus (Holmgr.)Al, Ab, N, Ka, Om. ibid.:30.

Echthronomas facialis (Thoms.) Ab, N. ibid.:31.

Olesicampe cavigena (Thoms.) Ab, N, Oa, Ks. ibid.:30.

- nigricoxa (Thoms.) Ab,N, Tb. ibid.:31.

- praeoccupator (Aub.) Le: Kilpisjärvi (V. Karvonen). ibid.:31.

- vitripennis (Holmgr.) Ab, Ob, Ks, Li. ibid.:31.

Lathrostizus flexicauda (Holmgr.) Le: Kilpisjärvi. Kopelke 1999 Cour. Forsch.inst. Senckenb.

212:37, 49 . 
- forticauda (Thoms.) Le: Kilpisjärvi. ibid.:54.

Eriborus perfidus (Grav.) (aberrans (Grav.)). Finland. Koponen \& al. 2003:43.

- terebrans (Grav.) Ab: Vihti (V. Karvonen). ibid.:31.

Ophion costatus Ratz. $N, \mathrm{Ka}$. ibid.:28.

- crassicornis Brock Ab, N, Ka,Ta. ibid.:28.

- forticornis Morl. Ab, N, Ka. ibid.:28.

- perkinsi Brock Ab, N, Ka, Sa. ibid.:28.

Enicospilus merdarius (Grav.) (sp. dist.) Finland. ibid.:34.

*Astiphromma peltolatum Schwenke Ab: “ Turkku, Peltola " (V.-M. Mukkala). Schwenke 2004 Entomofauna 25:83.

- simplex (Thoms.) (sp. dist.). Yu \& al. 2005:53969.

- splenium (Curt.) (plagiatum (Thoms.), strenuum (Holmgr.). ibid.:53970.

*Mesochorus oshobotrianus Schwenke Ob: "Oshobotria bor.” (K. Müller). Schwenke 2002 Entomofauna 23:88.

Exochus nigripalpis Thoms. Yu \& al. 2005:55344.

- incidens Thoms. (sp. dist.). ibid.:55286.

- septentrionalis Holmgr. (sp. dist.). ibid.:55417.

Agrypon anomelas (Grav.) Finland. Koponen \& al. 2003:35.

- batis (Ratz.) (stenostigma (Thoms.)) (sp. dist.) Finland. ibid.:35.

- canaliculatum (Ratz.) Ta: Urjala (T. Brander). ibid.:28.

! - hilaris (Tosq.) Kb: Ilomantsi. Humala 2001:208.

- rugifer (Thoms.) Al: Finström, Kb: Ilomantsi. Koponen \& al. 2003:28. Humala 2001:208.

Meteorus corax Marsh. Sa: Juva, Ristiina. Martikainen \& Koponen 2001 EF 12:170.

*Peristenus varisae Acht. $N$ : Helsinki, Viikki (A.-L. Varis). Varis \& van Achterberg 2001 Zool. Meded. 75:375.

Diospilus oleraceus Hal. (sp.dist.). Belokobylskij \& al. 2003 Beitr. Entomol. 53:384.

Bassus arcuatus (Reinh.) (sp.dist.). ibid.:355.

Microchelonus erosus (Herr.-Sch.) (sp.dist.) ibid.:375.

Chelonus humilis Thoms. (sp. dist.). Yu \& al. 2005:11031.

* Opius (Odontopoea) epulatiformis Fisch. N: Sibbo (A. Albrecht). Fischer 2001 Linz. Biol. Beitr. 33:10.

Opius (Utetes) posticatae Fisch. (hilaris Fisch., seebensteinensis Fisch.). Belokobylskij \& al. 2003:396.

* Opius (Phaedrotoma) recondens (Acht.) (reconditor auct. nec Wesm.). Finland. van Achterberg 2004 Zool. Meded. Leiden 78:344.

*Phaenocarpa ungulosetosa Acht. \& Kop. Al, N, St, $\mathrm{Ta}, \mathrm{Sa}, \mathrm{Om}, \mathrm{Ok}$. van Achterberg \& Koponen 2003 Zool. Meded. 77:293.

- angustiptera Papp Al, Ab, N, Ta, Sa, Kl, Ob, Ks,
$L k$. Koponen \& Vikberg 2005 EF 16:58.

* - hirsuta Kop. \& Vikb. Om: Kalajoki (M. Koponen). ibid.:52.

- picinervis (Hal.) Al, Ab, N, Ka, St, Ta, Sa, Kl, Tb, $\mathrm{Kb}, \mathrm{Om}$. ibid.:56.

Coelinius parvulus (Nees) (anceps (Curt.), circulator (Grav.)). Belokobylskij \& al. 2003:360.

Colastes incertus (Wesm.) (sp.dist.). ibid.:383.

Aleiodes linearis Hellén (sp.dist.). ibid.:398.

Bracon claripennis Thoms. Finland. Papp 2000 Ann. hist.-nat. Mus. Natn. Hungar. 92:242.

- pallicarpus Thoms. (sp.dist.) Finland. ibid.:256.

Neuroterus numismalis (Geoffr.) Ab, N. Roslin, Silfverberg \& Huldén 2004 Sahlbergia 8:100.

Copidosoma serricorne (Dalm.) Finland. Guerreri \& Noyes 2005 Syst. Ent. 30:111.

- sosares (Walk.) Finland. ibid.:118.

- cyaneum Hoff. Finland. ibid.:123.

- dius (Walk.) Finland. ibid.:126.

- genale (Thoms.) Finland. ibid.:130.

- radnense Erdös Finland. ibid.:135.

- bolivari Merc. Finland. ibid.:137.

- albipes (Westw.) Finland. ibid.:139.

- fuscisquama (Thoms.) Finland. ibid.:140.

- tibiale Hoff. Finland. ibid.:147

* - charon Guerr. \& Noy. Ab: Vichtis (W. Hellén), $N$ : Tvärminne (W. Hellén). ibid.:147.

- iracundum Erdös Finland. ibid.:152.

- anceus (Walk.) Finland. ibid.:154.

* Proclithrophorus genalis Vikb. \& Kop. Ob: Pudasjärvi (M. Koponen). Vikberg \& Koponen 2001 EF 12:177.

Phaenoserphus chittii (Morl.) (dubiosus Nixon) Kb: Ilomantsi. Humala 2001:205.

Macrohynnis lepidus Mayr N, St, Ta, Sb, Li. Vikberg \& Koponen 2002 EF 13:52.

- fragilis (Nixon) $K b$ : Tohmajärvi, Liperi. ibid.:52.

Allotropa conventus Manev. Ab: Västanfjärd, $N$ : Nurmijärvi. Buhl \& Koponen 2003 Ent. Fenn. 14:98.

Fidiobia polita Buhl Sb: Jäppilä (P.Martikainen). ibid.:99.

Platystasius antennatus (Sundh.) Sb: Savonranta (P.Martikainen). ibid.:99.

Pseudaphanomerus hyalinatus Szel. Sb: Savonranta (P.Martikainen). ibid.:99.

*Anopedias notaulatus Buhl $N$ : Nurmijärvi (M. Koponen), Ta: Juupajoki (M. Koponen). Buhl 2005 EF 16:74.

Inostemma acuminatum Toms. Lk: Kittilä. Buhl \& Koponen 2003:100.

* - koponeni Buhl N: Kirkkonummi (M. Koponen), $\mathrm{Sa}$ : Ristiina (M. Koponen), Kl: Parikkala (M. Koponen). Buhl 2005:75.

Acerotella evanescens (Kieff.) St: Vammala. Buhl \& Koponen 2003:99.

- humilis (Kieff.) N: Nurmijärvi. ibid.:99.

Aceroteta borealis Kozl. \& M. N: Nurmijärvi, Tuusula, Hyvinkää. ibid.:99. 
Isocybus bifracticornis (Zett.) Ok: Ristijärvi, Paltamo. ibid.:100.

- matuta (Walk.) Ok: Paltamo. ibid.:100.

- ocellaris Kieff. (cf.) Ta: Janakkala. ibid.:100.

* Platygaster anopediana Buhl Kb: Liperi (M. Koponen). Buhl 2005:78.

- attenuata Walk. N: Nurmijärvi. Buhl \& Koponen 2003:101.

- chloropus Thoms. $N$ : Nurmijärvi. ibid.:101.

- clavata Buhl Oa,Ok,Ob. ibid.:101.

- damokles (Buhl) Sa: Ristiina. ibid.:101.

- danielssoni Buhl Ta: Nastola. ibid.:101.

- demades Walk. N: Nurmijärvi. ibid.:101.

* - fennica Buhl Sb: Savonranta (P.Martikainen). Buhl 2003 Ent. Fenn. 14:110.

- gracilipes Hugg. N, St, Sa, Sb, Ok. Buhl \& Koponen 2003:101.

- hybrida Buhl $N$ : Nurmijärvi, Kb: Liperi. ibid.:102.

* - koponeni Buhl N: Nurmijärvi (M.Koponen). Buhl 2003:111.

- leptines Walk. Ok: Paltamo. Buhl \& Koponen 2003:102.

- longestriolatus Thoms. Sa: Ristiina. ibid.:102.

- lundensis Buhl N: Nurmijärvi. ibid.:102.

- lysicles Walk. $N$ : Nurmijärvi. ibid.:102.

- marginata Thoms. $N$ : Nurmijärvi. ibid.:102.

* - martikaineni Buhl Sb: Savonranta (P.Martikainen). Buhl 2003:112.

* - marttii Buhl $N$ : Nurmijärvi (M. Koponen), St: Vammala (M.Koponen). Buhl \& Koponen 2003.113; Buhl 2005:79.

- nisus Walk. N: Nurmijärvi. Buhl \& Koponen 2003:102.

- nottoni Buhl $N$ : Nurmijärvi, Sa: Ristiina. ibid.:102.

* - oblonga Buhl N: Nurmijärvi (M.Koponen). Buhl 2005:79.

- oscus Walk. Sa: Mikkeli. Buhl \& Koponen 2003:102.

- pedasus Walk. Ta: Nastola, Sa: Ristiina. ibid.:102.

- pelias Walk. $N$ : Nurmijärvi, Sa: Ristiina. ibid.:102.

- picipes Först. $N$ : Nurmijärvi. ibid.:103.

- rugosiceps Buhl N, Sb, Ob. ibid.:103.

- rutilipes Buhl $N$ : Helsinki. ibid.:103.

- sagana Walk. $N$ : Nurmijärvi. ibid.:103.

- soederlundi Buhl N: Nurmijärvi. ibid.:103.

- splendidula Ruthe N, Ta, Sa. ibid.:103.

- tisias Walk. (cf.) Sb: Savonranta, Ob: Tervola. ibid.: 103 .

- transversiceps Buhl N: Nurmijärvi. ibid.:103.

- vintheri Buhl Sb: Savonranta (P.Martikainen). ibid.: 103 .

Euxestonotus error (Fitch) St, Sb. ibid.:100.

- hasselbalchi Buhl $N$ : Nurmijärvi. ibid.:100.

Synopeas brevis Buhl $N$ : Nurmijärvi. ibid.:103.
- ciliatus Thoms. N: Nurmijärvi. ibid.:103.

* - discoideus Buhl N: Nurmijärvi (M. Koponen). buhl 2005:81.

- lugubris Thoms. Sb: Savonranta (P.Martikainen). Buhl \& Koponen 2003:104.

* - suomiana Buhl $N$ : Nurmijärvi (M.Koponen). Buhl 2003:116.

- trebius Walk. (cf.) Sb: Savonranta (P.Martikainen). Buhl \& Koponen 2003:104.

Leptacis breisteini Buhl $N$ : Nurmijärvi. ibid.:100.

- coryphe Buhl $N$ : Nurmijärvi. ibid.:100.

- orchymonti (Deb.) N: Nurmijärvi, Sa: Ristiina. ibid.: 100 .

- ozines (Walk.) N: Nurmijärvi, Ta: Hämeenlinna. ibid.: 100 .

- vlugi Buhl N: Nurmijärvi, Om: Alahärmä. ibid.: 101 .

Amblyaspis belus (Walk.) N, Ta, Sb, Ob. ibid.:99.

- scelionoides (Halid.) Sa: Ristiina. ibid.:99.

- tritici (Walk.) (cf.) Sb: Savonranta (P.Martikainen). ibid.:99.

Bocchus vernieri Olmi Ta: Tammela. Vikberg \& Koponen $2001 \mathrm{EF}$ 12:34.

Gonatopus horvathi Kieff. Ab: Lojo (W. Hellén), Sa: Joutseno (W. Hellén), Kl: Parikkala (W. Hellén). ibid.:35.

Bethylus berlandi Arlé Ab-Ok. Söderman \& Vikberg 2003:43.

Laelius pedatus (Say) $N, \mathrm{Ka}, \mathrm{Ok}, \mathrm{Ob}$. ibid.:43.

* - borealis Vikb. Al, Ab, N, Ka, Ta, Sa, Sb. Vikberg \& Koponen 2005 EF 16:29.

* - parcepilosus Vikb. Ta: Hämeenlinna, Sa: Mikkeli, $K l$ : Parikkala. ibid.:38.

* - virilis Vikb. Ta, Sa. ibid.:32.

Chrysis subcoriacea Linsenm. (sp.dist.) Al-Ob. Söderman \& Vikberg 2003:45.

Lasius psammophilus Seif. Ab-Ob. ibid.:46

- platythorax Seif. Ab-Ob. ibid.:46.

Priocnemis gracilis Haupt St: Punkalaidun. ibid.:60.

Evagetes alamannicus (Blüthg.) Al-Li. ibid.:49.

- subglaber (Haupt) Kb. ibid.:49.

Anoplius aeruginosus (Tourn.) Al-Ob. ibid.:49.

Arachnospila virgilabnormis (Wolf) Ab, N. ibid.:49.

Passaloecus turionum Dahlb. (sp.dist.). ibid.:51.

Ectemnius sexcinctus (F.) Ab: Bromarv (P. \& S.-L. Nuorteva), ibid.:62

Hylaeus difformis (Eversm.) Ab: Halikko (L. Lindgren \& J. Pöyry). Söderman \& Vikberg 2003:62, Söderman \& Leinonen 2003:60.

- pfankuchi Alfk. Al: Hammarland. Söderman \& Vikberg 2003:62, Söderman \& Leinonen 2003:70.

Andrena helvola (L.) Al: Hammarland, Geta. Söderman \& Vikberg 2003:62, Söderman \& Leinonen 2003:121.

- nigroaenea (Kby.) Al, Ab, N. Söderman \& Vikberg 2003:62, Söderman \& Leinonen 2003:103.

Lasioglossum quadrinotatum (Kby.) Al: Sund (P. Val- 
tonen). Söderman \& Vikberg 2003:62, Söderman \& Leinonen 2003:145.

- subfulvicorne (Blüthg.) (sp. dist.) $S a, S b, O k$. Söderman \& Vikberg 2003:62, Söderman \& Leinonen 2003:152.

Stelis minima Schenck (sp.dist.) Al-Kb. Söderman \& Vikberg 2003:57.

Osmia hyperborea Tkalcú Le, Li. Söderman \& Vikberg 2003:62, Söderman \& Leinonen 2003:211.

Bombus patagiatus Nyl. $K b$ : Eno (G. Söderman). Söderman \& Vikberg 2003:63, Söderman \& Leinonen 2003:294.

- schrencki (Mor.) N: Pyhtää (G. Söderman). Söderman \& Vikberg 2003:63, Söderman \& Leinonen 2003:322.

Present number: $5,976-40+239=6,175$ spp.

\section{Discussion}

The Finnish insect fauna is generally well known. Still, there are certain groups where much re- mains still to be done. That is particularly the case within Diptera Nematocera and Hymenoptera Parasitica, to some degree also in part of Hemiptera. The additions during the study period have been particularly numerous in those groups, and will undoubtedly be so further on, too, as long as taxonomic and faunistic research continues. In many other orders, our knowledge is much more complete, taxonomic work may result in occasional changes, and intense field work will now and then bring out some previously overlooked species, but a considerable percentage of the new records will really mean that the species have spread to new areas. Whether they are just strays, or whether there is going to be a colonization, remains then to be seen. Particularly the orders Coleoptera and Lepidoptera have shown several cases of colonization during the last decades, some of them spontaneous, others assisted by international trade. 